\title{
Influence of domain size on direct numerical simulation of turbulent flow in a moderately curved concentric annular pipe
}

Cite as: Phys. Fluids 32, 065105 (2020); https://doi.org/10.1063/5.0003436

Submitted: 01 February 2020 . Accepted: 24 May 2020 . Published Online: 12 June 2020

Edris Bagheri (D), Bing-Chen Wang (D), and Zixuan Yang (D)
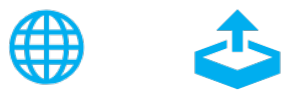

\section{ARTICLES YOU MAY BE INTERESTED IN}

Can a toilet promote virus transmission? From a fluid dynamics perspective

Physics of Fluids 32, 065107 (2020); https://doi.org/10.1063/5.0013318

Conditional spatially averaged turbulence and dispersion characteristics in flow over twodimensional dunes

Physics of Fluids 32, 065106 (2020); https://doi.org/10.1063/5.0008380

Vortex induced vibrations of wind turbine blades: Influence of the tip geometry

Physics of Fluids 32, 065104 (2020); https://doi.org/10.1063/5.0004005

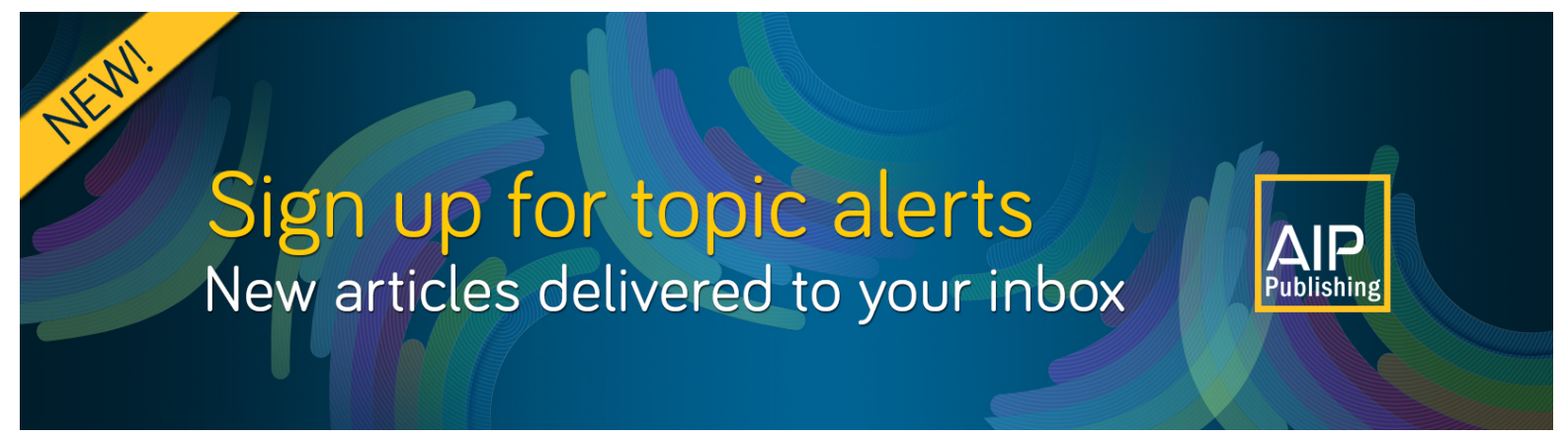




\title{
Influence of domain size on direct numerical simulation of turbulent flow in a moderately curved concentric annular pipe
}

\author{
Cite as: Phys. Fluids 32, 065105 (2020); doi: 10.1063/5.0003436 \\ Submitted: 1 February 2020 - Accepted: 24 May 2020 • \\ Published Online: 12 June 2020
}

Edris Bagheri,' (D) Bing-Chen Wang, ${ }^{1, a)}$ (D) and Zixuan Yang ${ }^{1,}$

AFFILIATIONS

1 Department of Mechanical Engineering, University of Manitoba, Winnipeg, Manitoba R3T 5V6, Canada

${ }^{2}$ State Key Laboratory of Nonlinear Mechanics, Chinese Academy of Sciences, Beijing 100190, China

\begin{abstract}
a) Author to whom correspondence should be addressed: Bing Chen.Wang@Umanitoba.Ca. Tel.: +1(204) 474-9305. Fax: $+1(204)$ 275-7507
\end{abstract}

\begin{abstract}
Direct numerical simulation (DNS) of turbulent flow in a concentric annular pipe was performed using a pseudo-spectral method computer code. In order to study the effects of computational domain size on the turbulence statistics, 12 test cases of different domain sizes are compared. The characteristics of the velocity field are examined at two different Reynolds numbers. It is observed that the predictive accuracy of the first- and second-order statistics is sensitive to the axial and azimuthal domain sizes. It is also found that the scales and dynamics of turbulence structures vary with the surface curvature of the concave and convex walls. The characteristic length scales of the turbulence structures are identified through a spectral analysis, and it is observed that a minimum computational domain is required in order to accurately capture the near-wall streaky and hairpin structures of a concentric annular pipe flow using DNS.
\end{abstract}

Published under license by AIP Publishing. https://doi.org/10.1063/5.0003436

\section{INTRODUCTION}

Fully developed turbulent concentric annular pipe flow driven by an axial pressure gradient is an intriguing topic with important applications in mechanical engineering. For instance, the physical processes related to the bypass air over a combustion chamber in a jet engine, removal of cutting material between a drill shaft and a well casing, and convection in a double-pipe heat exchanger are all dominated by turbulent flow through an annular passage. Owing to the difference in the transverse curvature of the inner and outer pipe walls, the spatial and temporal scales of turbulence are different near these two walls of a concentric annular pipe. This further results in an asymmetry in the velocity profile and difference in the scales of streaks and hairpin structures near the inner and outer cylinder surfaces. Thus, the flow physics in a concentric annular pipe are more complex than that of a round pipe or plane-channel flow. Furthermore, the case of concentric annular pipe flow is general because both plane-channel and round pipe flows are special cases of concentric annular pipe flows under the condition of $R_{i} / R_{o} \rightarrow 1$ and $R_{i} / R_{o}$ $\rightarrow 0$, respectively. Here, $R_{i}$ and $R_{o}$ (with $0 \leq R_{i} \leq R_{o}$ ) are the radii of the inner and outer pipes, respectively. The current research focuses on Poiseuille-type turbulent concentric annular pipe flow driven by a constant axial pressure gradient, which is different from the classical Taylor-Couette flow driven by wall shear due to relative rotation of the inner and outer cylinder surfaces.

Thus far, previous experimental and numerical studies of Poiseuille-type concentric annular pipe flow have primarily focused on the characteristics of the first- and second-order flow statistics (such as the locations corresponding to the maximum mean velocity and the zero mean Reynolds shear stress studied by Knudsen and Katz, ${ }^{4}$ Brighton and Jones, ${ }^{5}$ Rehme, ${ }^{6}$ Nouri et al., ${ }^{7}$ and Chung et $\left.a l .{ }^{8}\right)$. In the current literature, there has been a disagreement on whether the radial position corresponding to the maximum velocity collocates with that of the zero mean shear stress. The experiments of Knudsen and Katz ${ }^{4}$ and Brighton and Jones ${ }^{5}$ on concentric annular pipe water flows indicated that the maximum velocity and the zero mean shear stress coincide. However, Rehme ${ }^{6}$ reported that the radial position of the zero mean shear stress is closer to the 
inner wall than that of the maximum velocity based on their measurements of annular concentric pipe airflows using a double Pitot tube and hot-wire anemometry. Nouri et al. ${ }^{7}$ conducted comprehensive laser-Doppler velocimetry (LDV) measurements of the flow fields in both concentric and eccentric annuli for both Newtonian and non-Newtonian fluids. They showed that the radial positions of the maximum velocity and zero shear stress are not collocated. This contradiction is also seen among numerical simulations. Chung et al. ${ }^{8}$ performed direct numerical simulation (DNS) of a concentric pipe flow at Reynolds number $R e_{D_{h}}=U_{b} D_{h} / v=8900$, similar to the experimental conditions of Nouri et al., and later, Chung and Sung extended their DNS study to also include turbulent heat transfer under a constant wall heat flux ratio. Here, $U_{b}$ represents the bulk mean velocity, $D_{h}$ is the hydraulic diameter, and $v$ is the kinematic viscosity of the fluid. The DNS result of Chung et al. ${ }^{8}$ indicated that the deviation in the radial positions of the maximum mean axial velocity and zero shear stress is less than $1 \%$. However, a more recent DNS study of Boersma and Breugem ${ }^{10}$ (conducted under conditions of $R_{i} / R_{o}=0.1$ and $R e_{D_{h}}=8900-13940$ ) further showed the collocation of these two positions. As the first objective of this research, we aim at clearing the inconsistency of literature on this issue by proving analytically that the radial positions of the maximum velocity and zero shear stress are, in fact, strictly collocated, and we will also validate our analytical result using the DNS data compiled.

Over the past three decades, various DNS studies were performed to understand circular pipe flows and structures, which feature two-dimensional (2D) statistical homogeneity over an axialazimuthal cylindrical surface. In the homogeneous directions, typically, periodical boundary conditions are implemented. The minimum computational domain size over which a periodic boundary condition can be used for conducting DNS with sufficient predictive accuracy is a critical issue. In their pioneering DNS study of the minimum computational domain for near-wall turbulence, Jiménez and Moin $^{11}$ revealed that the prediction of turbulence statistics would not be accurate if the size of the computational domain is smaller than that of the so-called "minimal channel." Chin et al. ${ }^{12}$ performed DNS to investigate the influence of computational domain size on the first- and second-order turbulence statistics and axial velocity spectrum of turbulent circular pipe flows. They applied periodic boundary conditions to the axial and azimuthal directions for a wide range of pipe lengths for $L_{z}=\pi R-20 \pi R$, where $R$ is the radius of the circular pipe. In their DNS study of a turbulent plane Couette flow, Avsarkison et al. ${ }^{13}$ kept a computational domain length at $L_{z}$ $=20 \pi \delta$ to ensure capture of streamwise-elongated flow structures. Here, $\delta$ is one-half the plane channel height. Very recently, Yang and Wang ${ }^{14}$ conducted a DNS study of streamwise rotating turbulent channel flow at a low Reynolds number of $R e_{\tau}=u_{\tau} \delta / v=180$ in conjunction with a wide range of rotation numbers. They discovered that the wavelength of the streamwise-elongated Taylor-Görtler-like (TG-like) structures increases significantly with the rotation number. At their highest rotation number tested, an extremely long domain of $L_{z}=512 \pi \delta$ was used in order to correctly capture TG-like vortices.

Although there are many DNS investigations into the effects of computational domain size on the predictive accuracy of channel and pipe flows (see, for example, the work of Jiménez and Moin ${ }^{11}$ and Chin et $a .^{12}$ ), the number of systematic studies on the proper computational domain size for transient simulation of turbulent concentric annular pipe flow is still very limited in the current literature. In the DNS studies of Chung et al. ${ }^{8}$ and Boersma and Breugem, ${ }^{10}$ the concentric pipe length was set to $L_{z}=6 \pi \delta$ and $10 R_{o}$, respectively, based on an analysis of the two-point correlation coefficient of axial velocity fluctuations. Here, $\delta$ is one-half the cylinder gap, i.e., $\delta=\left(R_{o}-R_{i}\right) / 2$, defined in analogy to the usual convention for a plane-channel flow (in which one-half the channel height between two planes is often used as a basic measurement length scale). Quadrio and Luchini ${ }^{15}$ performed DNS study of turbulent concentric annular pipe flow, with the radius ratio kept at $R_{i} / R_{o}=0.33$ and 0.5 , and the axial pipe length set to $L_{z}=4 \pi \delta$. They examined the first- and second-order flow statistics and studied the effect of the transverse curvature on the peak value of Reynolds shear stresses. As the second objective of this research, we aim at conducting a systematic study of the minimum computational domain for DNS of concentric pipe flow in both physical and spectral spaces. We will show evidence that a much longer concentric pipe is needed in order to obtain accurate DNS results of flow statistics. To this purpose, a comparative study based on different domain sizes in the axial and azimuthal directions is conducted. The largest computational domain size $\left(L_{z}=30 \pi \delta\right.$ and $\left.L_{\theta}=2 \pi\right)$ tested here far exceeds those reported in the literature. Furthermore, based on the aforementioned investigation of the minimum computational domain size, highly accurate DNS datasets are compiled and used for studying turbulence statistical moments and coherent structures of the concentric annular pipe flow, which is the third objective of this research.

The remainder of this paper is organized as follows: in Sec. II, the test cases and the numerical algorithm for solving the governing equations are described. In Sec. III, the DNS results in the physical space are analyzed, including the first-, second-, third-, and fourth-order statistical moments of the velocity field, and the two-point correlation functions of velocity fluctuations. In Sec. IV, turbulent coherent structures are investigated and their scales are studied based on calculation of both two-dimensional (2D) and one-dimensional (1D) energy spectra. The scales of energetic eddies and the corresponding domain size for capturing them are examined through analyses conducted in both physical and spectral spaces. Finally, in Sec. V, major findings of this research are summarized.

\section{TEST CASE AND NUMERICAL ALGORITHM}

Figure 1 shows the schematic diagram and coordinate system of the test case, where $z, r$, and $\theta$ denote the axial, radial, and azimuthal coordinates, respectively. The velocity components corresponding to these three directions are represented by $u_{z}, u_{r}$, and $u_{\theta}$, respectively. In this paper, tensor notations are also used, and these three velocity components are denoted as $u_{1}, u_{2}$, and $u_{3}$, respectively. The equations that govern an incompressible flow with respect to a cylindrical coordinate system read

$$
\nabla \cdot \vec{u}=0
$$

$$
\frac{\partial \vec{u}}{\partial t}+\vec{u} \cdot \nabla \vec{u}=-\frac{1}{\rho} \nabla p+v \nabla^{2} \vec{u}-\frac{\Pi}{\rho} \widehat{\mathbf{e}}_{z},
$$




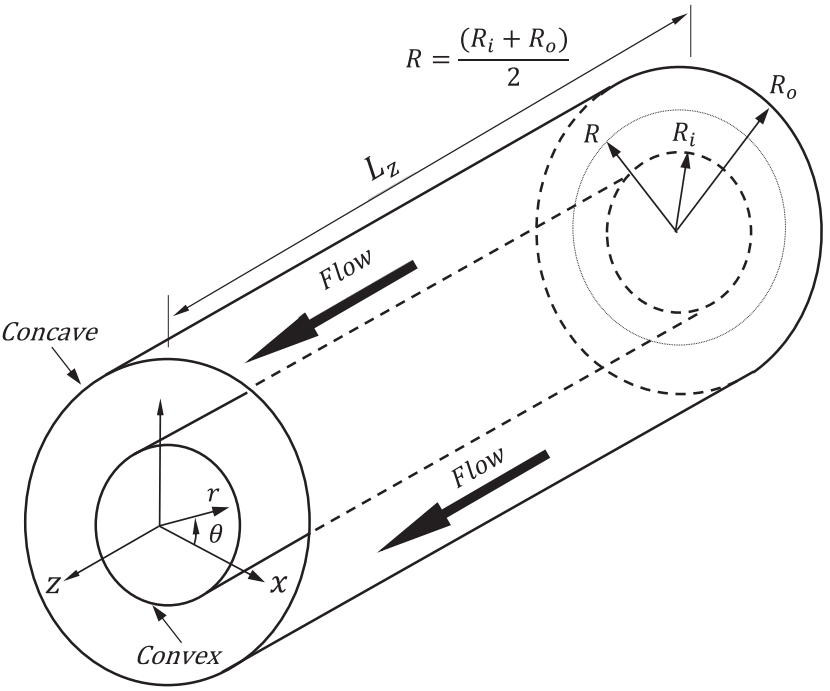

FIG. 1. Computational domain and coordinate system.

where $p, \rho$, and $v$ denote the pressure, density, and kinematic viscosity, respectively. $\Pi$ is the constant mean axial pressure gradient that drives the flow, and $\widehat{\mathbf{e}}_{z}$ is the base unit vector of the $z$-direction, with $\left|\widehat{\mathbf{e}}_{z}\right| \equiv 1$.

Table I summarizes the computational domain sizes and grid resolutions for the 14 test cases of two nominal Reynolds numbers of $R e_{D_{h}}=8900$ and 17700 . In order to facilitate our comparative study of the domain size effect on the predictive accuracy of flow statistics and coherent structures, the radius ratio is kept at $R_{i} / R_{o}=0.5$ for all 14 test cases. This radius ratio and lower nominal Reynolds number $\left(R e_{D_{h}}=8900\right)$ considered here are identical to those used in the work of Chung et al. ${ }^{8}$ and Nouri et al. ${ }^{7}$ The test cases are categorized and labeled using four initial letters: A, B, C, and D. Six different axial domain sizes with a full cross-sectional domain (referred to as the A-series test cases) are compared to examine the influence of axial computational domain size on the predictive accuracy of turbulent statistics in both physical and spectral spaces. In these six test cases, the axial domain size varies from $L_{z}=\pi \delta$ to $30 \pi \delta$, while the azimuthal domain size is fixed to $L_{\theta}=2 \pi$. The reason that a full azimuthal domain size is used in the A-series test cases is that this can completely shield off the effect of the azimuthal domain size on the flow statistics. The next group is the five B-series test cases, which examine the influence of azimuthal domain size $L_{\theta}$ on turbulent statistics. Five different azimuthal domain sizes ranging from $L_{\theta}$ $=\pi / 6$ to $2 \pi$ are compared. For the five B-series test cases, the axial domain size is fixed at $L_{z}=12 \pi \delta$ based on the conclusion obtained from the above comparative study of the A-series test cases. Case A4 (of A-series) is also case B5 (of B-series). For the purpose of comparison and code validation, the computational domain size of Chung et $a .^{8}$ is also considered, which leads to the C-series test cases. In Table I, our DNS run with a finer radial grid resolution is labeled as case $\mathrm{C}$ and that of Chung et al. ${ }^{8}$ is referred to as case Ca. In order to study the Reynolds number effect on the statistical moments of the velocity field, a higher nominal Reynolds number of $R e_{D_{h}}=17700$ is also considered, which is referred to as case D.

An in-house computer code developed based on the pseudospectral method was used for performing DNS, which was written using the FORTRAN 90/95 programming language. Message passing interface (MPI) libraries were employed to parallelize the computing processes. The advection terms were treated explicitly with a third-order temporal accuracy, whereas the pressure and diffusion terms were treated implicitly. The continuity constraint was enforced strictly at every stage of the iterative process by using the pressure-correction method. The viscous forces were decoupled by changing variables following Blackburn and Sherwin ${ }^{16}$. In order to expand the velocity and pressure fields, Fourier series were applied to the axial and azimuthal directions and Lagrange polynomials were used in the wall-normal direction. Aliasing errors were removed by using the $3 / 2$ rule. Periodic boundary conditions were applied to the axial and azimuthal directions, and the no-slip boundary condition was imposed on all solid surfaces. Equally spaced grid points were

TABLE I. Summary of test cases and grid resolutions. The nominal Reynolds number is $R e_{D_{h}}=8900$ for the A-, B-, and C-series test cases and is $R e_{D_{h}}=17700$ for case $D$.

\begin{tabular}{|c|c|c|c|c|c|c|c|}
\hline Cases & $L_{z} \times L_{\theta} \times L_{r}$ & $N_{z} \times N_{\theta} \times N_{r}$ & $\Delta z^{+}$ & $\left(R_{i} \Delta \theta\right)^{+}$ & $\left(R_{o} \Delta \theta\right)^{+}$ & $\Delta r_{\min }^{+}$ & $\Delta r_{\max }^{+}$ \\
\hline A1 & $\pi \delta \times 2 \pi \times 2 \delta$ & $36 \times 512 \times 64$ & 12.819 & 3.607 & 7.214 & 0.180 & 7.376 \\
\hline A2 & $2 \pi \delta \times 2 \pi \times 2 \delta$ & $64 \times 512 \times 64$ & 14.528 & 3.634 & 7.267 & 0.182 & 7.431 \\
\hline A3 & $6 \pi \delta \times 2 \pi \times 2 \delta$ & $192 \times 512 \times 64$ & 14.422 & 3.607 & 7.215 & 0.180 & 7.377 \\
\hline A4 (B5) & $12 \pi \delta \times 2 \pi \times 2 \delta$ & $384 \times 512 \times 64$ & 14.390 & 3.599 & 7.198 & 0.180 & 7.360 \\
\hline A5 & $18 \pi \delta \times 2 \pi \times 2 \delta$ & $580 \times 512 \times 64$ & 14.361 & 3.617 & 7.234 & 0.181 & 7.396 \\
\hline A6 & $30 \pi \delta \times 2 \pi \times 2 \delta$ & $960 \times 512 \times 64$ & 14.461 & 3.617 & 7.234 & 0.181 & 7.396 \\
\hline B1 & $12 \pi \delta \times \pi / 6 \times 2 \delta$ & $384 \times 42 \times 64$ & 14.679 & 3.730 & 7.459 & 0.184 & 7.508 \\
\hline B2 & $12 \pi \delta \times \pi / 4 \times 2 \delta$ & $384 \times 64 \times 64$ & 14.489 & 3.624 & 7.248 & 0.181 & 7.411 \\
\hline B3 & $12 \pi \delta \times \pi / 2 \times 2 \delta$ & $384 \times 128 \times 64$ & 14.475 & 3.621 & 7.241 & 0.181 & 7.404 \\
\hline B4 & $12 \pi \delta \times 3 \pi / 4 \times 2 \delta$ & $384 \times 192 \times 64$ & 14.431 & 3.609 & 7.219 & 0.181 & 7.381 \\
\hline $\mathrm{C}$ & $6 \pi \delta \times \pi / 2 \times 2 \delta$ & $192 \times 128 \times 64$ & 14.422 & 3.607 & 7.215 & 0.180 & 7.377 \\
\hline $\mathrm{Ca}$ (Chung et al.) & $6 \pi \delta \times \pi / 2 \times 2 \delta$ & $192 \times 128 \times 65$ & 14.30 & 3.62 & 7.11 & 0.12 & 12.96 \\
\hline $\mathrm{D}$ & $8 \pi \delta \times \pi / 2 \times 2 \delta$ & $500 \times 280 \times 144$ & 13.878 & 3.099 & 6.199 & 0.069 & 6.100 \\
\hline
\end{tabular}




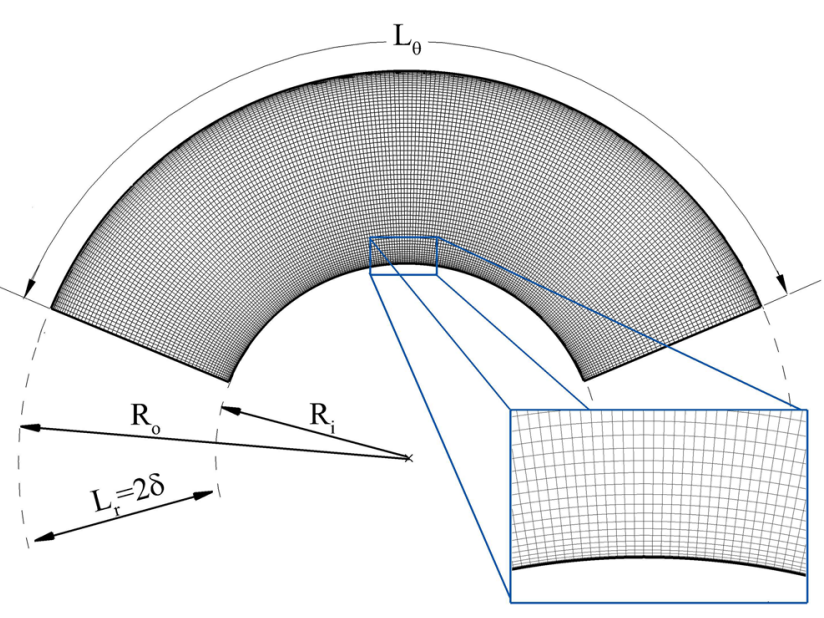

FIG. 2. Cross-sectional view of the mesh for case B4.

used in both axial and azimuthal directions, and Chebyshev-GaussLobatto points were used in the radial direction for improving the spatial resolution near the wall. As shown in Table I, in all our 12 test cases of $\mathrm{A}, \mathrm{B}$, and $\mathrm{C}$ series, the axial grid resolution ranges from $\Delta z^{+}=12.819$ for case A1 to 14.679 for case B1 and the azimuthal grid resolutions range from $\left(R_{i} \Delta \theta\right)^{+}=3.599$ for case A4 (or B5) to $\left(R_{o} \Delta \theta\right)^{+}=7.459$ for case B1. From Table I, it is clear that the axial and azimuthal grid resolutions are close to those used in the work of Chung et al. ${ }^{8}$ The grid resolution of case D is slightly finer than those of the other test cases in general. As an example for showing the grid system, Fig. 2 provides a cross-sectional view of the mesh used for performing DNS of case B4. The finest radial grid resolution for the first node off the wall ranges from $\Delta r_{\text {min }}^{+}=0.180$ to 0.184 , which is slightly larger than that used in the work of Chung et al. ${ }^{8}$ but satisfies the need for performing rigorous DNS of wall-bounded turbulence. More importantly, the maximum radial grid resolution in our DNS is restricted to $\Delta r_{\max }^{+}=7.376-7.508$, which is much finer than that $\left(\Delta r_{\text {max }}^{+}=12.96\right)$ used in the work of Chung et al. ${ }^{8}$ In Table I, the wall unit is defined based on the kinematic viscosity $v$ of the fluid and averaged wall friction velocity $u_{\tau}=\sqrt{\tau_{w} / \rho}$, where $\tau_{w}$ is the mean wall shear stress based on weighted averaging over the concave and convex walls, i.e.,

$$
\tau_{w}=\frac{R_{i} \tau_{w i}+R_{o} \tau_{w o}}{R_{i}+R_{o}} .
$$

The grid resolutions listed in Table I are calculated based on the averaged wall friction velocity $u_{\tau}$. The wall frictional velocity can also be defined locally as $u_{\tau i}=\sqrt{\tau_{w i} / \rho}$ and $u_{\tau o}=\sqrt{\tau_{w o} / \rho}$, with $\tau_{w i}=\rho v\left(d\left\langle u_{z}\right\rangle / d r\right)_{r=R_{i}}$ and $\tau_{w o}=-\rho v\left(d\left\langle u_{z}\right\rangle / d r\right)_{r=R_{o}}$ at the inner and outer cylinder walls, respectively. The values of $u_{\tau i}$ and $u_{\tau o}$ are different due to the surface curvature of the inner and outer cylinders. The ratio between these two wall friction velocities is $u_{\tau i} / u_{\tau o}$ $=1.0596$ under the test conditions. In this paper, unless otherwise noted, we use the local wall friction velocities $u_{\tau i}$ and $u_{\tau o}$ in the non-dimensionalization of various quantities.

In our analysis, an instantaneous flow variable (for example, $u_{z}$ ) is decomposed into an averaged and a fluctuating component, i.e., $u_{z}=\left\langle u_{z}\right\rangle+u_{z}^{\prime}$, where a pair of angular brackets $\langle\cdot\rangle$ represent temporal and spatial averaging over the homogeneous directions. Specifically, the spatial averaging is performed over a $z-\theta$ cylindrical surface at an arbitrary radial position $r$, and temporal averaging was performed over 35 large-eddy turnover times (LETOTs). Here, one LETOT is defined as $\delta / u_{\tau}$, which is a measure of the required time for large-scale structures with scale of $\delta$ to be uncorrelated. According to Adrian, ${ }^{18}$ the length scale of large-scale motions (or "turbulent bulges") is approximately $2-3 \delta$ in a wall-bounded flow. Thus, an extended duration of 35 LETOTS facilitates the evolution of large-scale structures and achieving good convergence in the calculation of the statistics of the velocity field. The computational time step was kept at $0.001 \delta / U_{b}$ to keep the Courant-FriedrichsLewy (CFL) number less than 0.8 . All computations were performed on the WestGrid (Western Canada Research Grid) supercomputers. Furthermore, spectral accuracy was also ensured during the computation of flow statistics.

Table II lists the mean flow parameters calculated from DNS for different test cases. Here, Reynolds numbers $R e_{\tau_{i}}=u_{\tau i} \delta_{t i} / v$ and $R e_{\tau_{o}}=u_{\tau o} \delta_{t o} / v$ are defined based on the wall friction velocities $\left(u_{\tau i}\right.$ and $u_{\tau o}$ ) and boundary layer thicknesses $\left(\delta_{t i}\right.$ and $\delta_{t o}$ ) over the inner and outer cylinder walls, respectively. The boundary layer thickness, $\delta_{t}$, is the wall-normal distance across a boundary layer from either the inner or the outer wall to the point where the mean axial velocity reaches its maximum (correspondingly, the Reynolds shear stress is zero). The exact wall-normal position of this point will be investigated separately in Sec. III B. The numerical simulations were set up under the condition of a constant mean streamwise pressure gradient, which can be determined as

$$
\Pi=-\frac{2\left(\tau_{w i} R_{i}+\tau_{w o} R_{o}\right)}{\left(R_{o}^{2}-R_{i}^{2}\right)} .
$$

In the above equation, the values of $\tau_{w i}$ and $\tau_{w o}$ can be further determined based on the values of the skin friction coefficients $\left(C_{f i}\right.$ and $C_{f_{o}}$ ) at the inner and the outer cylinder walls, respectively. Two nominal Reynolds numbers are tested, i.e., $R e_{D_{h}}=8900$ and 17700 . In order to set up the numerical simulation, we used the skin friction coefficient values given in the DNS study of Chung et al. ${ }^{8}$

TABLE II. Mean flow parameters calculated from DNS.

\begin{tabular}{lccccc}
\hline \hline Cases & $\delta_{t i} / \delta$ & $\delta_{t o} / \delta$ & $R e_{\tau_{i}}$ & $R e_{\tau_{o}}$ & $R e_{D_{h}}$ \\
\hline $\mathrm{A} 1$ & 0.867 & 1.133 & 130.91 & 164.24 & 8944 \\
$\mathrm{~A} 2$ & 0.887 & 1.113 & 136.86 & 161.29 & 8953 \\
$\mathrm{~A} 3$ & 0.880 & 1.120 & 134.14 & 161.55 & 8926 \\
$\mathrm{~A} 4$ (B5) & 0.877 & 1.123 & 133.12 & 161.79 & 8935 \\
$\mathrm{~A} 5$ & 0.880 & 1.120 & 134.30 & 162.15 & 8926 \\
$\mathrm{~A} 6$ & 0.881 & 1.119 & 134.69 & 161.82 & 8917 \\
$\mathrm{~B} 1$ & 0.893 & 1.107 & 139.77 & 161.75 & 9042 \\
$\mathrm{~B} 2$ & 0.878 & 1.122 & 134.25 & 162.71 & 8962 \\
$\mathrm{~B} 3$ & 0.877 & 1.123 & 133.89 & 162.75 & 8944 \\
$\mathrm{~B} 4$ & 0.879 & 1.121 & 133.82 & 161.98 & 8944 \\
$\mathrm{C}$ & 0.880 & 1.120 & 134.14 & 161.55 & 8935 \\
$\mathrm{D}$ & 0.882 & 1.118 & 253.02 & 302.87 & 17770 \\
\hline \hline
\end{tabular}


for the lower Reynolds number case of $R e_{D_{h}}=8900$, which is $C_{f i}$ $=0.00941$ and $C_{f_{o}}=0.00849$ at the inner and outer cylinder walls, respectively. From Eq. (4), it is straightforward that the value of the mean streamwise pressure gradient can be alternatively determined as $\Pi=-C_{f} \rho U_{b}^{2} /\left(R_{o}-R_{i}\right)$, based on the averaged friction coefficient defined as $C_{f}=C_{f i} R_{i} /\left(R_{i}+R_{o}\right)+C_{f_{o}} R_{o} /\left(R_{i}+R_{o}\right)$. The value of $C_{f}$ can be determined from the empirical equation given by Nouri et al., i.e., $C_{f}=0.36\left(R e_{D_{h}}\right)^{-0.39}$. We used this method to set up the numerical simulation for the higher Reynolds number case of $R e_{D_{h}}=17700$. Test runs were conducted, and minor adjustments to the value of $\Pi$ were made to ensure that the value of $R e_{D_{h}}$ calculated from DNS converges to its nominal value within a reasonable range of error tolerance. As is clear in Table II, the values of $R e_{D_{h}}$ calculated from DNS of cases of relatively large domains (such as cases A3-A6, B3, B4, C, and D) are satisfactory, which have a very small error of less than $0.4 \%$ in comparison with the nominal Reynolds values (of $R e_{D_{h}}=8900$ and 17700 ). However, the values of $R e_{D_{h}}$ calculated from DNS of cases of small domain sizes (such as cases $\mathrm{A} 1, \mathrm{~A} 2, \mathrm{~B} 1$, and B2) are less satisfactory. The values of $R e_{D_{h}}$ and $U_{b}$ calculated from the DNS are determined by the mean velocity field, and the influence of domain size on the predictive accuracy of DNS on the mean velocity field will be examined systematically in Sec. III.

\section{TURBULENCE STATISTICS IN PHYSICAL SPACE}

\section{A. Mean velocity}

Figure 3(a) compares the mean velocity profiles of A-, B-, C-, and D-series test cases along with the DNS data of Chung et al. ${ }^{8}$ under the testing conditions of two nominal Reynolds numbers of $R e_{D_{h}}=8900$ and 17700 . To facilitate a fair comparison between the convex and concave sides, the mean axial velocity is plotted in the "global coordinate" (non-dimensionalized by one-half the cylinder gap $\delta)$, i.e., $(r-R) / \delta$, and scaled based on the mean friction velocity $u_{\tau}$. The distributions of the mean axial velocity are asymmetric in the radial direction, which is a distinctive feature of a concentric annular pipe flow. As is evident in Fig. 3(a), for the lower nominal Reynolds number cases, the velocity profiles collapse well for cases A2-A6 and B2-B4. An excellent agreement between the results of case $\mathrm{C}$ and the reference data of case $\mathrm{Ca}$ is observed, which confirms the predictive accuracy of the computer code in terms of the generation of reliable DNS data of the mean velocity of the concentric turbulent annular pipe flow. However, from Fig. 3(a), it is seen that the value of the mean axial velocity $\left\langle u_{z}\right\rangle^{+}$is slightly overpredicted by about $2.7 \%$ in case $\mathrm{A} 1$ and slightly underpredicted by $1.3 \%$ in case B1, respectively. As shown in Table I, case A1 has the smallest axial domain size (which is $L_{z}=\pi \delta$ ) and case B1 has the smallest azimuthal domain size (which is $L_{\theta}=\pi / 6$ ). This indicates that if we solely focus on the mean velocity profiles and accept an arbitrary error tolerance up to $3 \%$, it would be very tempting to conclude that the axial and azimuthal domain sizes $L_{z}=\pi \delta$ and $L_{\theta}=\pi / 6$ are sufficient for conducting DNS of the current concentric annular pipe flow. In other words, the criterion based on the predictive accuracy of the first-order statistical moment of the velocity field can be very tolerant, and literally, all 12 test cases of the lower nominal Reynolds number can well predict the value of $\left\langle u_{z}\right\rangle^{+}$. However, in the sections to be followed, it will be shown that this conclusion is, in fact, incorrect with respect to the prediction of the secondorder statistical moments and spectral analysis of the velocity field.

It should be indicated that the above observations were made by investigating the effects of the axial and azimuthal domain sizes independently through A- and B-series test cases. The reason that case A1 fails is that its domain size $L_{z}=\pi \delta$ is too small to capture the characteristic axial length scales of flow structures, and similarly, case B1 fails because its azimuthal domain size is too small to capture the characteristic azimuthal length scales of flow structures. Therefore, the performance of a combined case of $\pi \delta \times \pi / 6$ $\times 2 \delta$ is expected to be even worse as it is too small to capture flow structures in both axial and azimuthal directions. Later in Sec. IV, concrete evidence will be provided to support this physical analysis. We will explain rigorously why these two small axial and azimuthal domain sizes $\left(L_{z}=\pi \delta\right.$ and $\left.L_{\theta}=\pi / 6\right)$ fail to capture the characteristic wavelengths of the most energetic eddies, either independently or in combination, through analyses of $1 \mathrm{D}$ and $2 \mathrm{D}$ premultiplied spectra of turbulence.

Figure 3(b) compares the profiles of the mean axial velocity on the convex and concave sides of cases A6 and D for two different nominal Reynolds numbers of $R e_{D_{h}}=8900$ and 17 700. Among the 12 A-, B-, and C-series test cases for the lower nominal Reynolds number, case $A$ has the longest axial domain size of $L_{z}=30 \pi \delta$ and a full azimuthal domain size of $L_{\theta}=2 \pi$. As such, the influence of the axial domain size on the numerical results is the minimum, and

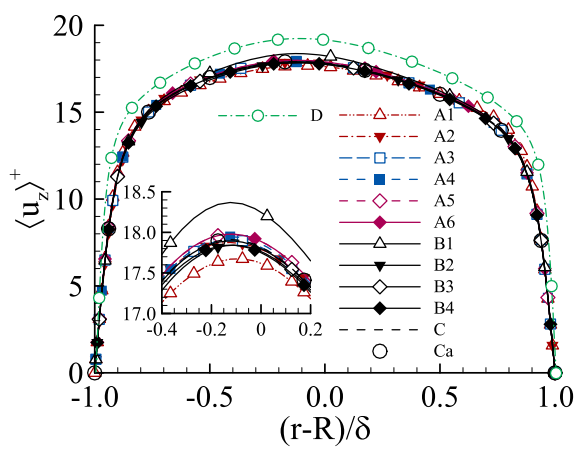

(a)

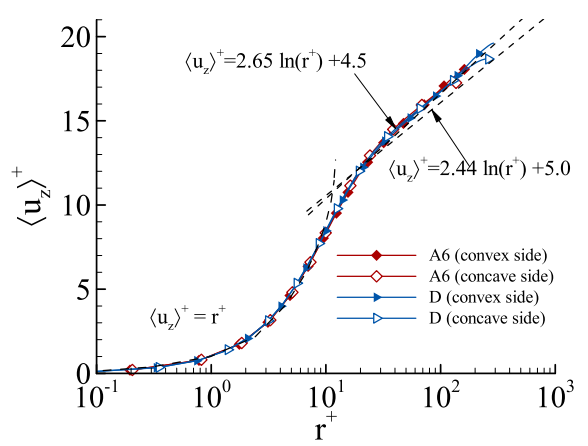

(b)
FIG. 3. Profiles of the mean axial velocity $\left\langle u_{z}\right\rangle^{+}$. (a) In global coordinate for A-, $\mathrm{B}-, \mathrm{C}$-, and $\mathrm{D}$-series test cases and (b) in wall coordinate for cases $A 6$ and $D$. To facilitate the comparison of cases of the lower nominal Reynolds number, their profiles are partially enlarged and shown in an inset graph in (a). 
the influence from the azimuthal domain size is completely shielded off. The exceptionally large computational domain of case A6 is also the largest in the current literature for concentric annular pipe flows, which is five times that used by Chung et al. ${ }^{8}$ under a similar testing condition. The DNS result of the velocity field is the most accurate in case A6, and here, we use this test case to demonstrate the transverse curvature effect on the wall scaling of the mean axial velocity profile. To this purpose, the mean axial velocity and wall coordinates are scaled based on the local friction velocities of the convex $\left(u_{\tau i}\right)$ and concave $\left(u_{\tau o}\right)$ walls in Fig. $3(\mathrm{~b})$. The profile of the standard law-of-the-wall based on von Kármán's two-layer model for a zero-pressure-gradient (ZPG) boundary layer over a flat plate is also displayed for the purpose of comparison.

From Fig. 3(a), it is observed that as the nominal Reynolds number increases from $R e_{D_{h}}=8900$ to 17700 , the magnitude of $\left\langle u_{z}\right\rangle^{+}$increases slightly when they are displayed in the global coordinate $(r-R) / \delta$. However, the profiles of $\left\langle u_{z}\right\rangle^{+}$of these two Reynolds numbers collapse once they are plotted in wall coordinate $r^{+}$in Fig. 3(b). Apparently, the velocity magnitudes on both concave and convex sides of the concentric pipe are slightly larger than that prescribed by the classical log-law for a ZPG boundary layer over a flat plate, $\left\langle u_{z}\right\rangle^{+}=2.44 \ln \left(r^{+}\right)+5.0$. Furthermore, through a linear regression analysis, it is observed that the velocity profiles at two Reynolds numbers collapse well on a log-law of $\left\langle u_{z}\right\rangle^{+}=2.65 \ln \left(r^{+}\right)$ +4.5 . Owing to the curvature difference between the two cylinder surfaces, the mean velocity profile of concentric annular pipe flow is asymmetrical in the radial direction [Fig. 3(a)], which leads to differences in the velocity profiles plotted in the wall coordinates measured from the convex and concave walls in Fig. 3(b). From
Fig. 3(b), it is clear that the differences are the most apparent in the channel center. This is because not only the values of $u_{\tau i}$ and $u_{\tau o}$ are different (with $u_{\tau i} / u_{\tau o}=1.057$ ), but also the peak position of $\left\langle u_{z}\right\rangle^{+}$ deviates from the domain center of $(r-R) / \delta=0$. Specifically, from Fig. 3(a) and Table II, it is observed that the profile of $\left\langle u_{z}\right\rangle^{+}$peaks at $(r-R) / \delta=-0.119$. In the following, the mechanism underlying the shift of the peak position of $\left\langle u_{z}\right\rangle^{+}$toward the inner cylinder wall will be further analyzed from the point of view of the balance of viscous and turbulent shear stresses.

\section{B. Reynolds stresses}

Figures 4 and 5 show the profiles of all four non-trivial Reynolds stresses at two nominal Reynolds numbers of $R e_{D_{h}}=8900$ and 17700 (including the reference case $\mathrm{Ca}$ ) in the global coordinate. In order to facilitate a clear comparison of all 14 test cases under the influences of different axial and azimuthal domain sizes and Reynolds numbers, the results of A-series test cases are compared with those of $\mathrm{C}$ - and D-series test cases in Fig. 4, while those of B-series test cases are compared with those of C-and D-series test cases in Fig. 5. All Reynolds stresses have been non-dimensionalized by the mean wall friction velocity $\left(u_{\tau}\right)$. From Figs. 4 and 5 , it is apparent that the profiles of all Reynolds normal and shear components are asymmetrical in the radial direction at both Reynolds numbers. By comparing the results of cases $\mathrm{C}$ and $\mathrm{Ca}$, it is evident that in either Fig. 4 or Fig. 5, the profiles of Reynolds normal and shear stresses obtained in the current DNS agree well with those of Chung et al. ${ }^{8}$ In Fig. 4, the Reynolds stress profiles of the A-series test cases collapse, except for cases A1 and A2. As is shown in Fig. 4(a),

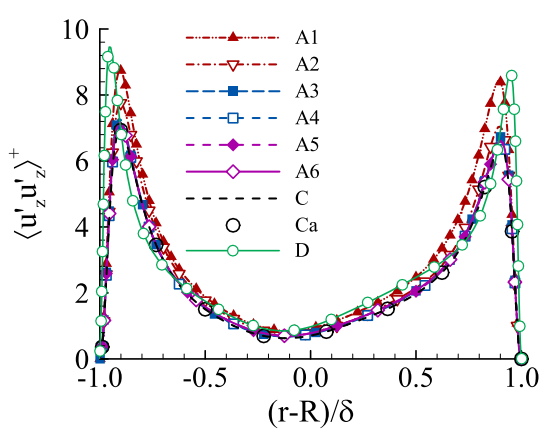

(a)

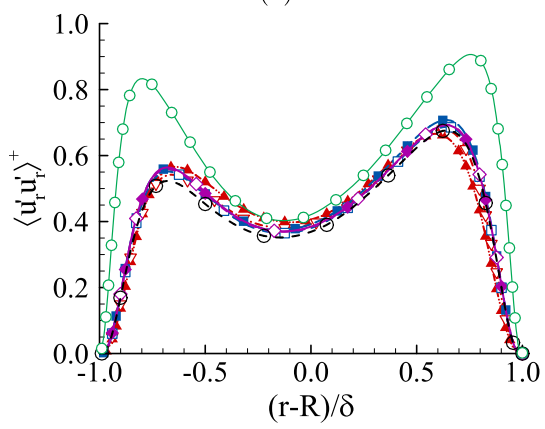

(c)

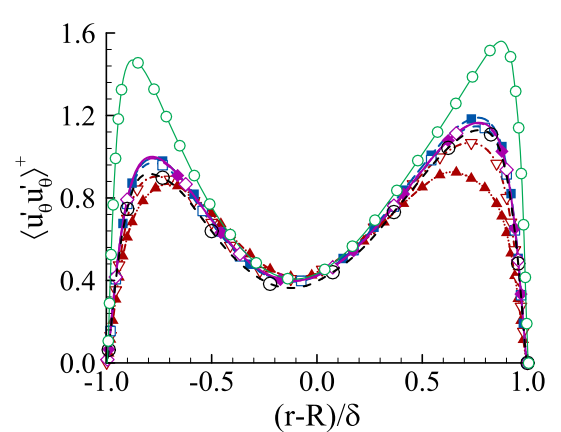

(b)

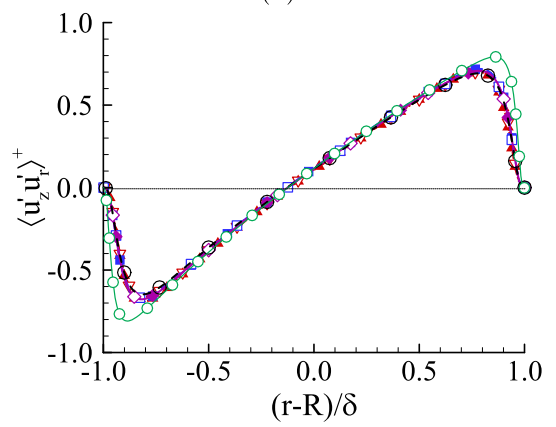

(d)
FIG. 4. Profiles of Reynolds stresses $\left\langle u_{i}^{\prime} u_{j}^{\prime}\right\rangle^{+}$predicted based on various computational domain sizes of the Aseries test cases in comparison with those of the $\mathrm{C}$ - and D-series test cases: (a) $\left\langle u_{z}^{\prime} u_{z}^{\prime}\right\rangle^{+}$, (b) $\left\langle u_{\theta}^{\prime} u_{\theta}^{\prime}\right\rangle^{+}$, (c) $\left\langle u_{r}^{\prime} u_{r}^{\prime}\right\rangle^{+}$, and (d) $\left\langle u_{z}^{\prime} u_{r}^{\prime}\right\rangle^{+}$ 


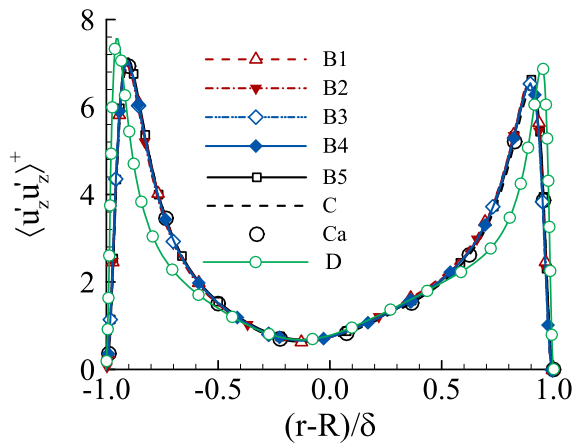

(a)

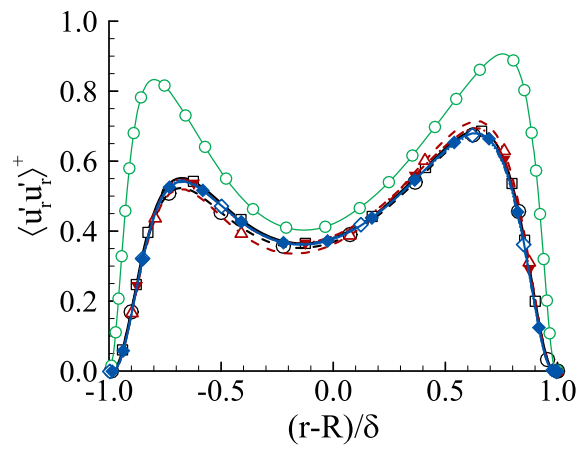

(c)

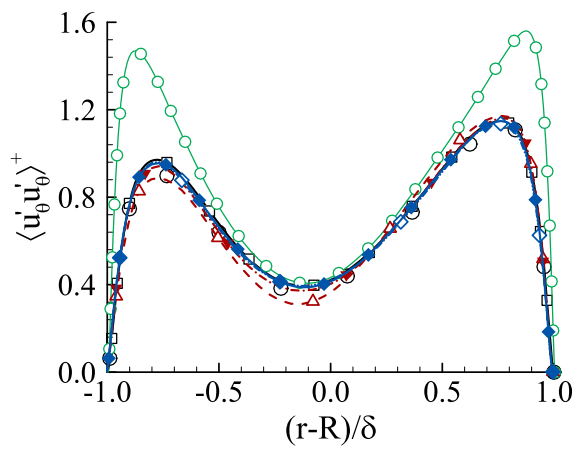

(b)

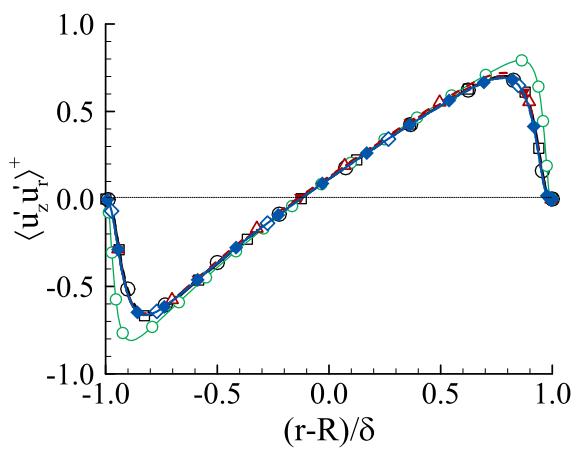

(d)
FIG. 5. Profiles of Reynolds stresses $\left\langle u_{i}^{\prime} u_{j}^{\prime}\right\rangle^{+}$predicted based on various computational domain sizes of the Bseries test cases in comparison with those of the $\mathrm{C}$ - and D-series test cases: (a) $\left\langle u_{z}^{\prime} u_{z}^{\prime}\right\rangle^{+}$, (b) $\left\langle u_{\theta}^{\prime} u_{\theta}^{\prime}\right\rangle^{+}$, (c) $\left\langle u_{r}^{\prime} u_{r}^{\prime}\right\rangle^{+}$ and (d) $\left\langle u_{z}^{\prime} u_{r}^{\prime}\right\rangle^{+}$ the magnitudes of the axial Reynolds normal stress of cases A1 and A2 are over-predicted in the near-wall region on both sides of the concentric annular pipe. Meanwhile, Fig. 4(b) shows that in cases $\mathrm{A} 1$ and $\mathrm{A} 2$, not only the value of $\left\langle u_{\theta}^{\prime} u_{\theta}^{\prime}\right\rangle^{+}$is underpredicted, but also the locations of the near-wall peaks on both sides of the concentric annular pipe are incorrectly predicted, which shift toward the center of the channel. In view of this, it concluded that in order to correctly predict the Reynolds stress level, the minimal axial computational domain size must be kept at $L_{z}=6 \pi \delta$ (or approximately, $L_{z}^{+}=2800$ ). The influence of azimuthal computational domain size on the predictive accuracy of Reynolds stresses can be identified by comparing the DNS results of the five B-series cases with those of cases $\mathrm{C}$ and $\mathrm{Ca}$ at a lower nominal Reynolds number of $R e_{D_{h}}=8900$ in Fig. 5. From Fig. 5(a), it is observed that the prediction of the profile of $\left\langle u_{z}^{\prime} u_{z}^{\prime}\right\rangle^{+}$is satisfactory, which is in an excellent agreement with the results of cases $\mathrm{C}$ and $\mathrm{Ca}$ in all five $\mathrm{B}$-series cases. However, as is clear from Figs. 5(b) and 5(c), the values of $\left\langle u_{\theta}^{\prime} u_{\theta}^{\prime}\right\rangle^{+}$and $\left\langle u_{r}^{\prime} u_{r}^{\prime}\right\rangle^{+}$of case B1 are underpredicted on the convex side and in the central region of the concentric annular passage. Clearly, based on the prediction of Reynolds stresses, the minimal azimuthal computational size must be stretched from $L_{\theta}=\pi / 6$ (which is the previous conclusion based on the prediction of the mean axial velocity profiles shown in Fig. 3) to $L_{\theta}=\pi / 4$.

From Figs. 4 and 5, it is seen that the Reynolds number has a significant influence on the predicted value of Reynolds stresses. Clearly, as the nominal Reynolds number increases from $R e_{D_{h}}$ $=8900$ to 17700 , the magnitudes of $\left\langle u_{\theta}^{\prime} u_{\theta}^{\prime}\right\rangle^{+}$and $\left\langle u_{r}^{\prime} u_{r}^{\prime}\right\rangle^{+}$vary significantly. By contrast, those of $\left\langle u_{z}^{\prime} u_{z}^{\prime}\right\rangle^{+}$and $\left\langle u_{z}^{\prime} u_{r}^{\prime}\right\rangle^{+}$are less sensitive.
Furthermore, it is interesting to observe from both Figs. 4(d) and 5(d) that the Reynolds shear stress $\left\langle u_{z}^{\prime} u_{r}^{\prime}\right\rangle^{+}$is the least sensitive to the axial and azimuthal computational domain sizes. A further study of the transport equation of the mean axial velocity facilitates a better understanding of this invariant behavior of the Reynolds shear stress. Considering that the flow is statistically stationary and homogeneous in the $r-\theta$ cylindrical surface, the following equation that expresses the viscous and turbulent shear stresses can be derived from Eq. (2):

$$
\frac{\tau_{\text {tot }}}{\rho}=v \frac{d\left\langle u_{z}\right\rangle}{d r}-\left\langle u_{z}^{\prime} u_{r}^{\prime}\right\rangle=\frac{\Pi}{2 \rho} r+\frac{D}{r}
$$

where $\tau_{\text {tot }}$ denotes the total shear stress (as the summation of the viscous and turbulent shear stresses) and $D$ is a constant of integration given as

$$
D=\frac{R_{o}^{2} R_{i} u_{\tau i}^{2}+R_{i}^{2} R_{o} u_{\tau o}^{2}}{R_{o}^{2}-R_{i}^{2}} .
$$

From Eq. (5), it is straightforward that at the radial position of $r=r_{m}=\sqrt{-2 \rho D / \Pi}$, the total shear stress vanishes, i.e., $\tau_{\text {tot }}$ $=0$. Consequently, $v d\left\langle u_{z}\right\rangle / d r-\left\langle u_{z}^{\prime} u_{r}^{\prime}\right\rangle=0$. In other words, at radial position $r_{m}$, if $\left\langle u_{z}^{\prime} u_{r}^{\prime}\right\rangle=0$, then it is guaranteed that $d\left\langle u_{z}\right\rangle / d r=0$ or vice versa. From this analysis, it is clear that the radial position for zero Reynolds shear stress is strictly collocated with that for the maximum of mean velocity (where $d\left\langle u_{z}\right\rangle / d r=0$ ) at radial position $r_{m}$ in a concentric annular pipe flow. The above analytical approach for deriving this conclusion is not complex; however, it helps to clear 


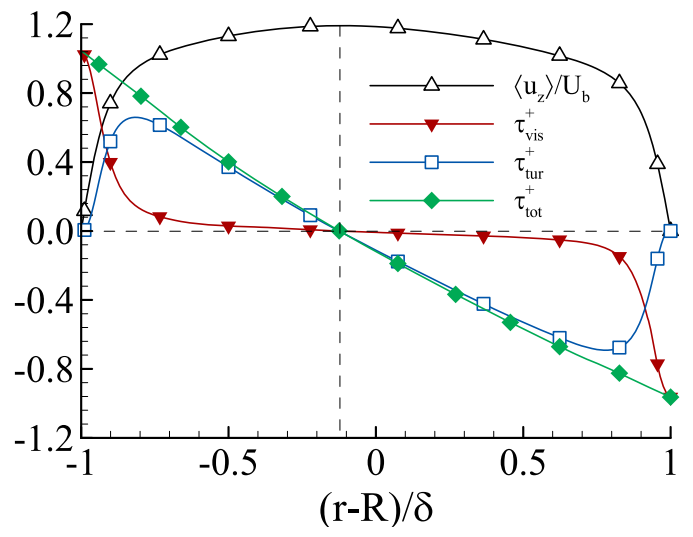

FIG. 6. Profiles of the mean axial velocity $\left\langle u_{z}\right\rangle$ and budget balance of total shear stress $\left(\tau_{\text {tot }}=\tau_{\text {vis }}+\tau_{\text {tur }}\right)$ displayed in the global coordinate based on the DNS results of case $A 6$. Considering the large differences in their absolute magnitudes, the mean axial velocity $\left\langle u_{z}\right\rangle$ is non-dimensionalized using the bulk mean velocity $U_{b}$, while the shear stresses are non-dimensionalized based on the mean wall friction velocity $u_{\tau}$

the disagreement on this issue seen in the literature (as reviewed in Sec. I). Now, we can say with certainty that the experimental observations of Knudsen and $\mathrm{Katz}^{4}$ and Brighton and Jones, ${ }^{5}$ and DNS results of Boersma and Breugem ${ }^{10}$ are accurate on this issue. The thicknesses of boundary layers developed over the inner and outer cylinder walls are different to reflect the difference in the surface curvature of these two walls, and their values can be determined as $\delta_{t i}=\delta+r_{m}$ and $\delta_{t o}=\delta-r_{m}$, respectively. The values of $\delta_{t i}$ and $\delta_{t o}$ calculated from DNS for all test cases are presented in Table II.

To further verify the above analysis using DNS, the shear stress balance of case A6 (which has the largest computational domain) is shown in Fig. 6. Figure 6 shows the profiles of the mean axial velocity, viscous Reynolds shear stress $\tau_{\text {vis }} / \rho=v d\left\langle u_{z}\right\rangle / d r$, turbulent Reynolds shear stress $\tau_{\text {tur }} / \rho=-\left\langle u_{z}^{\prime} u_{r}^{\prime}\right\rangle$, and total Reynolds shear stress $\tau_{\text {tot }}=\tau_{\text {vis }}+\tau_{\text {tur. }}$. In Fig. 6, all shear stress terms have been non-dimensionalized using the mean friction velocity. Clearly, at the radial position demarcated by the black dashed line, the axial mean velocity $\left\langle u_{z}\right\rangle$ reaches its maximum, and Reynolds shear stress $\left\langle u_{z}^{\prime} u_{r}^{\prime}\right\rangle$ becomes zero concurrently. Specifically, at the lower nominal Reynolds number $R e_{D_{h}}=8900$, the radial position where $\tau_{\text {tot }}^{+}=0$, $\left\langle u_{z}^{\prime} u_{r}^{\prime}\right\rangle^{+}=0$, and $d\left\langle u_{z}\right\rangle / d r=0$ deviates from the domain center and occurs instead at $(r-R) / \delta=-0.119$. Furthermore, from Table II, it can be inferred that this special radial position remains almost unchanged as the nominal Reynolds number increases to $R e_{D_{h}}=17700$, which is located at $(r-R) / \delta=-0.118$.

\section{Higher-order statistics}

Thus far, the effects of surface curvature and Reynolds number on the turbulent flow field have been examined through the first- and second-order flow statistics. In order to develop a deeper understanding of these effects on the transport of momentum, the third- and fourth-order statistical moments of the velocity field can be examined. Because the higher-order statistical moments of the velocity field are more sensitive than the lower-order ones in the calculations, we restrict our examination of the domain size effects on the predictive accuracy of DNS to the study of the first- and second-order statistical moments (e.g., mean velocity, Reynolds stresses, two-point correlations, and energy spectra) in both physical and spectral spaces, which represents a usual practice in the literature on the study of the minimum domains for DNS. ${ }^{11,14}$ In the following, we focus our attention to the physics of the flow while we study the properties of the skewness and flatness factors. Specifically, we intend to examine the Reynolds number effects on the skewness and flatness factors by comparing their values of cases A6 and D; and furthermore, we study the curvature effects on the skewness and flatness factors by comparing their values on the convex and concave sides of the concentric annular pipe. The velocity skewness and flatness factors are defined as

$$
S\left(u_{i}^{\prime}\right)=\frac{\left\langle u_{i}^{\prime 3}\right\rangle}{\left\langle u_{i}^{\prime 2}\right\rangle^{3 / 2}} \quad \text { and } \quad F\left(u_{i}^{\prime}\right)=\frac{\left\langle u_{i}^{\prime 4}\right\rangle}{\left\langle u_{i}^{\prime 2}\right\rangle^{2}},
$$

respectively. Figures 7 and 8 compare the skewness and flatness factors of all three velocity fluctuating components at two nominal Reynolds numbers of $R e_{D_{h}}=8900$ and 17700 through cases A6 and $\mathrm{D}$.

For turbulence signals obeying the ideal Gaussian distribution, $S\left(u_{i}^{\prime}\right) \equiv 0$. From Fig. 7 , it is clear that the value of the skewness factor of azimuthal velocity fluctuations follows the Gaussian distribution [i.e., $S\left(u_{\theta}^{\prime}\right)=0$ ], which reflects the fact that flow is statistically axial-symmetric and, therefore, homogeneous in the azimuthal direction. However, the skewness factors of the other two components $\left[S\left(u_{z}^{\prime}\right)\right.$ and $\left.S\left(u_{r}^{\prime}\right)\right]$ of the velocity field deviate significantly from the Gaussian distribution as a result of wall anisotropy. As the nominal Reynolds number increases from $R e_{D_{h}}=8900$ to 17700 , the amplitude of $S\left(u_{r}^{\prime}\right)$ varies little, but that of $S\left(u_{z}^{\prime}\right)$ increases significantly.

The flow physics are dominated by sweeping and ejection events near both convex and concave cylinder walls, although the strengths of these events are different due to the difference in the surface curvature of the two cylinders. The ejection events are characterized by $S\left(u_{z}^{\prime}\right)<0$ and $S\left(u_{r}^{\prime}\right)>0$, while the sweeping events are characterized by $S\left(u_{z}^{\prime}\right)>0$ and $S\left(u_{r}^{\prime}\right)<0$. The ejection and sweeping events are associated with the so-called "Q2" and "Q4" events in the quadrant analysis of Reynolds stresses, respectively. ${ }^{18}$ The quadrant analysis of Reynolds shear stresses can be conducted with respect to the sign of $u_{i}^{\prime}$ or $u_{i}^{\prime 3}$. This is because $\operatorname{sign}\left(u_{i}^{\prime}\right)=\operatorname{sign}\left(u_{i}^{\prime 3}\right)$. Therefore, sweeping and ejection events of a wall-bounded flow can be studied based on the skewness factor. In order to facilitate a clear discussion of near-wall ejection of sweeping events, regions corresponding to these two events are delineated using vertical dashed lines, which go through the zero-crossing points of the skewness factors in the radial direction [at which $S\left(u_{z}^{\prime}\right)=0$ and $S\left(u_{r}^{\prime}\right)=0$ ]. The Reynolds number effects on the sweeping and ejection events are evident, which occur within $r^{+} \in[5,8]$ and $r^{+} \in[28,134]$ on the convex cylinder side but within $r^{+} \in[3,8]$ and $r^{+} \in[30,161]$ on the concave cylinder side, respectively, at the lower nominal Reynolds number of $R e_{D_{h}}=8900$. As the Reynolds number increases to $R e_{D_{h}}=17700$, the strengths of the ejection and sweeping events become stronger as the magnitudes of both $S\left(u_{z}^{\prime}\right)$ and $S\left(u_{r}^{\prime}\right)$ increase. At $R e_{D_{h}}=17700$, the sweeping and ejection events occur within $r^{+} \in[5,12]$ and $r^{+} \in[30,253]$ on the convex cylinder side but within $r^{+} \in[4,12]$ and $r^{+} \in[31,302]$ 


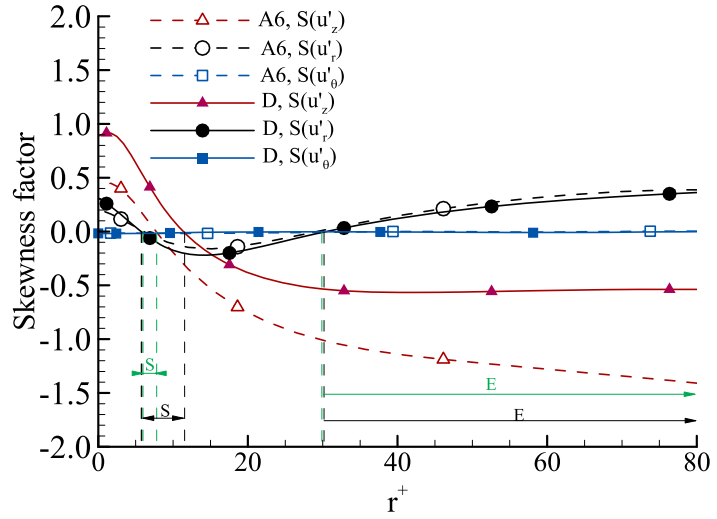

(a)

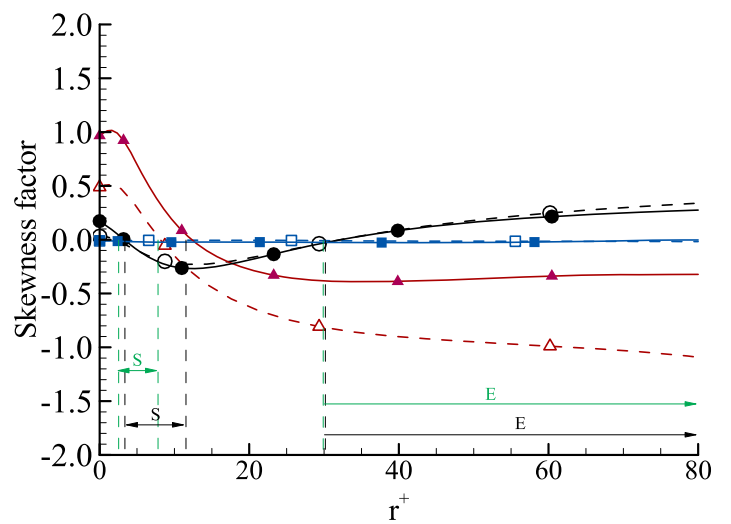

(b)

FIG. 7. Skewness factors of the velocity field on the (a) convex and (b) concave sides of the concentric annular pipe for cases $A 6$ and $D$. The vertical dashed lines demarcate the zero-crossing points of the skewness factors for $S\left(u_{z}^{\prime}\right)=0$ and $S\left(u_{r}^{\prime}\right)=0$. Near-wall regions featuring the sweeping and ejection events are labeled using "S" and "E," in either green or black color corresponding to either case A6 or D, respectively. The skewness factor of the ideal Gaussian distribution is also plotted using a thin dashed line, which is $S\left(u_{i}^{\prime}\right) \equiv 0$.

on the concave cylinder side, respectively. From these numbers, it is clear that the profiles of $S\left(u_{z}^{\prime}\right)$ and $S\left(u_{r}^{\prime}\right)$ are asymmetrical in the wall-normal direction as a result of the curvature difference between the convex and concave cylinder walls, a physical feature that is different from that of the classical 2-D plane-channel flows. ${ }^{17,19}$ In fact, this asymmetrical feature can be easily seen by directly comparing Figs. 7(a) and 7(b), which shows that the amplitudes of $S\left(u_{z}^{\prime}\right)$ and $S\left(u_{r}^{\prime}\right)$ in these two figures are different at the same radial position $r^{+}$.

From Fig. 8, it is clear that the flatness factors of all three components of velocity fluctuations peak at the wall, a pattern that is a characteristic of near-wall turbulence also observed in the classical turbulent plane-channel flows. ${ }^{17,19}$ The magnitude of $F\left(u_{i}^{\prime}\right)$ is the largest near the wall and gradually converges to $F\left(u_{i}^{\prime}\right)=3$ as the distance from wall increases, which is the theoretical value for the Gaussian distribution. The Reynolds number effects on the flatness factor is evident. As the nominal Reynolds number increases from $R e_{D_{h}}=8900$ to 17700 , the amplitude of $F\left(u_{\theta}^{\prime}\right)$ varies little, but those of $F\left(u_{r}^{\prime}\right)$ and $F\left(u_{z}^{\prime}\right)$ increase significantly. Furthermore, by comparing Figs. 8(a) and 8(b), it is clear that the magnitude of the $F\left(u_{i}^{\prime}\right)$ is larger near the concave side than near the convex side in general, as a result of the curvature difference between the inner and outer cylinder walls.

\section{Two-point correlation}

Two-point correlation is a conventional tool for studying the adequacy of the computational domain size as well as the length

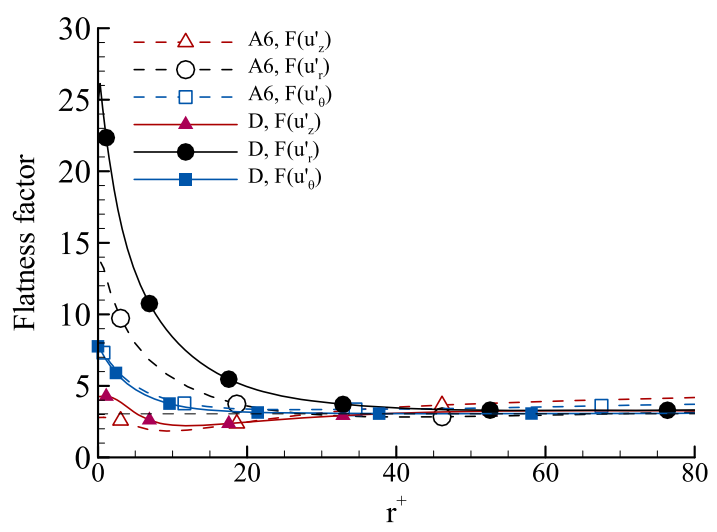

(a)

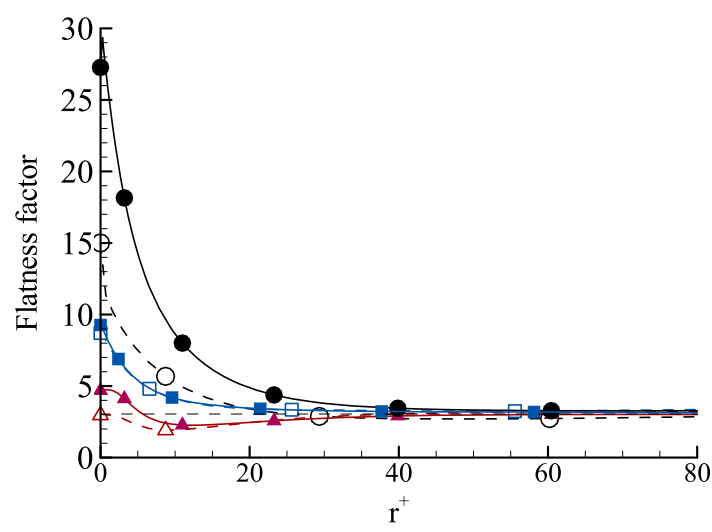

(b)

FIG. 8. Flatness factors of the velocity field on the (a) convex and (b) concave sides of the concentric annular pipe for cases $A 6$ and $D$. The flatness factor of the ideal Gaussian distribution is also plotted using a thin dashed line, which is $F\left(u_{i}^{\prime}\right) \equiv 3$. 
scales of coherent structures. The axial and azimuthal 1D two-point correlation functions for the axial velocity fluctuations are defined as

$$
R_{z z}(\Delta z)=\frac{\left\langle u_{z}^{\prime}(z, r \theta, r, t) u_{z}^{\prime}(z+\Delta z, r \theta, r, t)\right\rangle}{\left\langle u_{z}^{\prime}(z, r \theta, r, t) u_{z}^{\prime}(z, r \theta, r, t)\right\rangle}
$$

and

$$
R_{z z}(r \Delta \theta)=\frac{\left\langle u_{z}^{\prime}(z, r \theta, r, t) u_{z}^{\prime}(z, r \theta+r \Delta \theta, r, t)\right\rangle}{\left\langle u_{z}^{\prime}(z, r \theta, r, t) u_{z}^{\prime}(z, r \theta, r, t)\right\rangle}
$$

respectively.

Figures 9(a) and 9(b) compare the profiles of the axial 1D twopoint correlation coefficient $R_{z z}(\Delta z)$ of the A-series test cases calculated over the cylindrical surface positioned at $r^{+}=15$ from the convex and concave walls, where Reynolds normal stress $\left\langle u_{z}^{\prime} u_{z}^{\prime}\right\rangle^{+}$ reaches its peak value. Given their overly short axial domain sizes, the correlation coefficient curves of cases A1 and A2 end with finite values far above $R_{z z}(\Delta z)=0$, clearly suggesting insufficiency of the axial domain sizes to justify the use of the assumption of periodic boundary condition in these two test cases. By contrast, the twopoint correlation coefficients of cases A3-A6 fall to $R_{z z}(\Delta z)=0$ at $\Delta z^{+} \approx 1000$. This indicates that a minimum domain of $L_{z}=6 \pi \delta$ (of case A3) is, indeed, required to capture the length scale of axial turbulent flow structures. Figures 9 (c) and 9 (d) compare the azimuthal $1 \mathrm{D}$ two-point correlation $R_{z z}(r \Delta \theta)$ at $r^{+}=15$ on the convex and concave sides, respectively. The value of $R_{z z}(r \Delta \theta)$ becomes negative and reaches its minimum at $(r \Delta \theta)^{+} \approx 60$ and $(r \Delta \theta)^{+} \approx 90$ on the convex and concave sides, respectively. This indicates that the mean spacing between the near-wall streaks is smaller on the concave side and larger on the convex side due to the curvature difference between these two cylinder walls. The value of $R_{z z}(r \Delta \theta)$ does not end with zero in cases B1 and B2, suggesting that the azimuthal computational domain sizes are insufficient for accurately conducting DNS in these two cases. Based on the above analysis of the 1D two-point

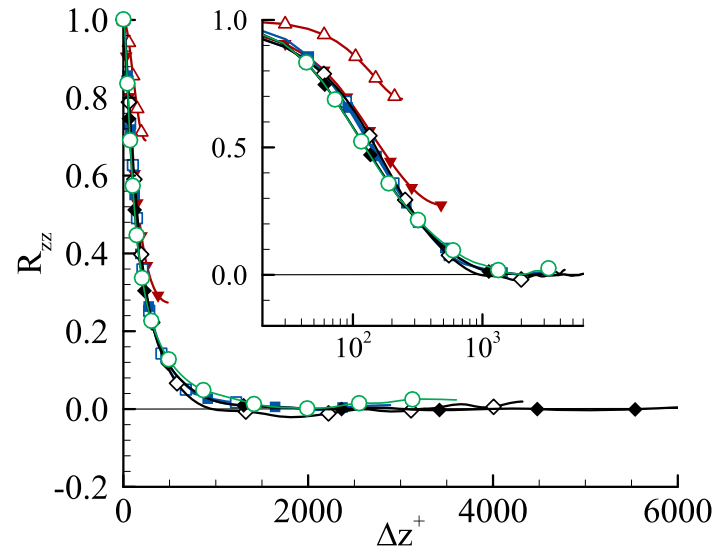

(a)

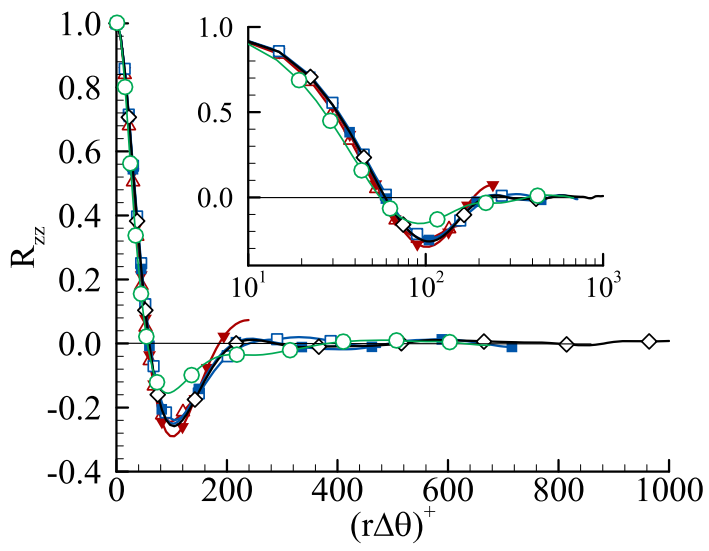

(c)

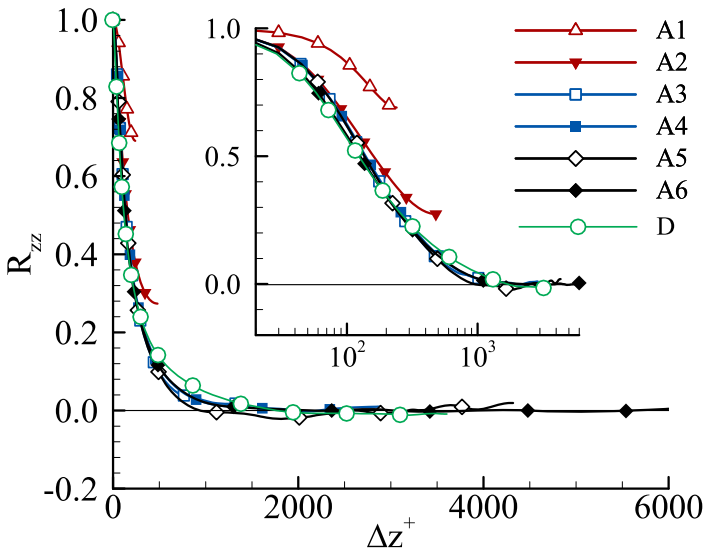

(b)

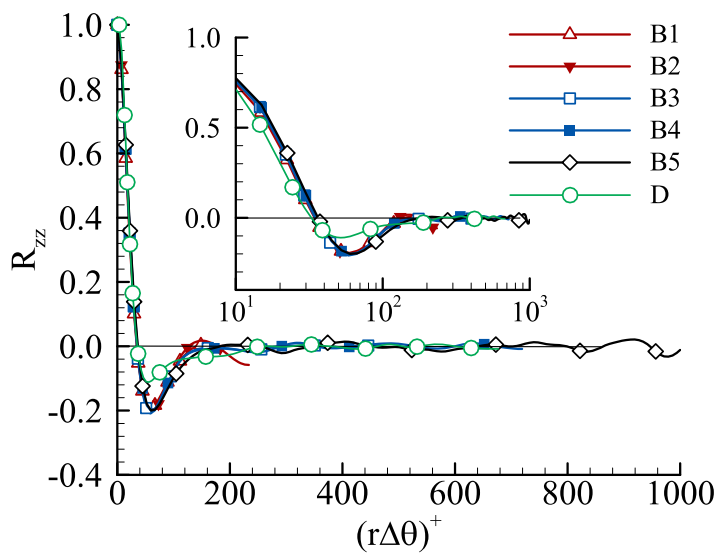

(d)

FIG. 9. Profiles of normalized two-point correlation coefficient $R_{z z}$ of the axial velocity fluctuations $\left(u_{z}^{\prime}\right)$ calculated over the cylindrical surface located at $r^{+}=15$ from the convex and concave walls, where the Reynolds normal stress $\left\langle u_{z}^{\prime} u_{z}^{\prime}\right\rangle$ reaches its peak value. (a) Axial correlations for A-and D-series test cases (convex side), (b) axial correlations for A- and D-series test cases (concave side), (c) azimuthal correlations for B- and D-series test cases (convex side), and (d) azimuthal correlations for B-and D-series test cases (concave side) 
correlation coefficients and previous analysis of the first- and second-order flow statistics, it is tempting to conclude that the minimum computational domain should be kept at $L_{z}=6 \pi \delta$ and $L_{\theta}$ $=\pi / 2$ in order to capture the length scales of energetic eddy motions at the lower nominal Reynolds number of $R e_{D_{h}}=8900$. This would lead to the conclusion that the domain of cases $\mathrm{C}$ and $\mathrm{Ca}$ is proper. However, so far, our study (conducted in Sec. III) has been limited exclusively to the physical space based on the analysis of the firstand second-order statistical moments of the velocity field and 1D two-point correlation coefficients, $R_{z z}(\Delta z)$ and $R_{z z}(r \Delta \theta)$. It should be noted that the choice of the computational domain sizes purely based on evidence in the physical space may not be conclusive. To confirm, the study needs to be further refined by examining the characteristic wavelengths of turbulent flow structures in the spectral space. In fact, we will show in Sec. IV that this suggested minimal domain (of $L_{z}=6 \pi \delta$ and $\theta=\pi / 2$ ) is actually insufficient, and a proper computational domain that allows for capturing the most energetic eddy motions is that of case B4 (of $L_{z}=12 \pi \delta$ and $L_{\theta}=3 \pi / 4$ ) based on the analysis of the axial and azimuthal 1D premultiplied spectra of velocity fluctuations.

\section{TURBULENCE STRUCTURES AND SPECTRAL ANALYSIS}

\section{A. Scales of hairpin structures and near-wall streaks}

In a concentric annular pipe flow, the scales of energetic eddy motions are dominated by two types of coherent turbulent structures, i.e., near-wall streaks and hairpin structures. The presence of near-wall streaks represents a universal feature of wall-bounded turbulent flows. Figure 10 shows the near-wall streaks on the convex and concave sides at $r^{+}=15$, where the maximum TKE occurs. The near-wall streaks are visualized using non-dimensionalized axial velocity fluctuations $u_{z}^{\prime+}$, which show a persistent and regular pattern. From Fig. 10, it is clear that low- and high-speed streaky structures alternate and are uniformly distributed in the axial and azimuthal directions. As one of the most energetic near-wall flow structures, these energy-containing streaks are elongated in the axial direction, which need to be captured in DNS by using a properly sized axial domain. If the axial domain is too short to capture the streaky structures, the axial length scales of the streaks will be artificially chopped off or distorted, and consequently, the level of TKE contained by near-wall streaks cannot be accurately calculated by DNS.

In Sec. III, we studied the minimum domain size required for properly conducting DNS in the physical space based on the analysis of a variety of flow statistics, including the two-point correction coefficients. As is well known, energy spectra are closely related to twopoint correction coefficients simply because they are counterparts of each other in Fourier transform. Different from two-point correlations, energy spectra can show precisely the turbulence energy level of flow structures at each specific wavelength, which, in turn, facilitates identifying the characteristic length scale (wavelength) of turbulence structures at an arbitrary turbulence energy level. The axial and azimuthal length scales of coherent structures can be examined precisely through the analysis of the premultiplied 2D energy spectrum, $k_{z} k_{\theta} \check{E}_{i i}$, where $\check{E}_{i i}=\check{E}_{i i}\left(k_{z}, k_{\theta}, r\right)$ is the $2 \mathrm{D}$ energy spectrum of velocity fluctuations in a homogeneous $r-\theta$ cylindrical surface, defined as

$$
\check{E}_{i i}\left(k_{z}, k_{\theta}, r\right)=2 \widehat{\widehat{u_{i}^{\prime}}\left(k_{z}, k_{\theta}, r\right){\widehat{u_{i}^{\prime}}}^{*}\left(k_{z}, k_{\theta}, r\right)}
$$

for $i=1,2$, or 3 (no summation convention implied). Here, an overline $\overline{(~)}$ indicates time averaging, $\widehat{u_{i}^{\prime}}$ represents a Fourier coefficient of $u_{i}^{\prime}, \widehat{u}_{i}^{*}$ denotes its complex conjugate, and $k_{z}$ and $k_{\theta}$ denote the axial and azimuthal wavenumbers, respectively. For discrete Fourier transform performed in a cylindrical coordinate system, these two wavenumbers are determined as $k_{z}=n_{z} k_{z 0}$ and $k_{\theta}=n_{\theta} k_{\theta 0}$ for $n_{z}$ $\in\left[-N_{z} / 2, N_{z} / 2-1\right]$ and $n_{\theta} \in\left[-N_{\theta} / 2, N_{\theta} / 2-1\right]$, respectively. Here,

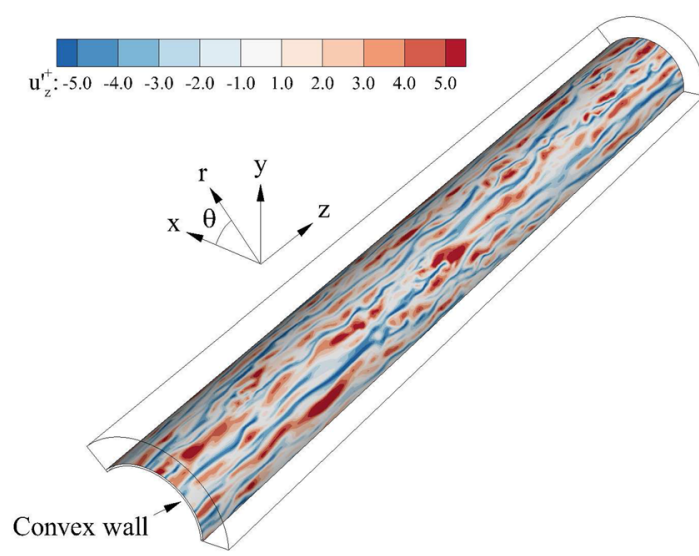

(a)

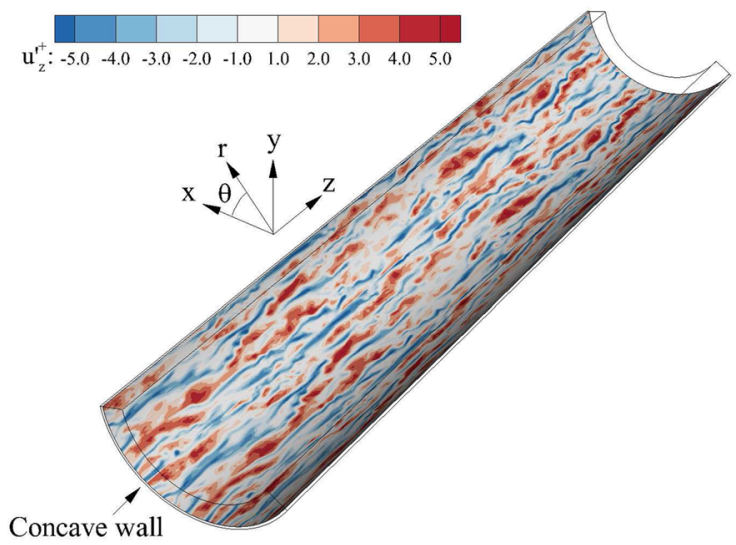

(b)

FIG. 10. Contours of the non-dimensionalized axial velocity fluctuations $u_{z}^{\prime+}$ of case B4, plotted at $r^{+}=15$ on the (a) convex and (b) concave sides, where the maximum TKE occurs. The contours are colored using the magnitude of the instantaneous axial velocity fluctuations $u_{z}^{\prime+}$. To enhance the visual clarity of near-wall structures, only a portion of the computational domain of case B4 is plotted. The plotted domain sizes are $L_{z}=6 \pi \delta$ and $L_{\theta}=\pi / 2$. 
$k_{z 0}=2 \pi / L_{z}$ and $k_{\theta 0}=2 \pi /\left(r \cdot L_{\theta}\right)$ are the lowest positive wavenumbers in the axial and azimuthal directions determined directly based on the domain sizes $L_{z}$ and $L_{\theta}$, respectively. The premultiplied 2D energy spectrum $k_{z} k_{\theta} \check{E}_{i i}$ is advantageous in identifying the characteristic wavenumbers $\left(k_{z}\right.$ and $\left.k_{\theta}\right)$ of the most energetic eddies in a $r-\theta$ cylindrical surface. In our discussion, besides wavenumbers, we also use wavelengths to evaluate the length scales, which are defined as $\lambda_{z}=2 \pi / k_{z}$ and $\lambda_{\theta}=2 \pi / k_{\theta}$. Clearly, both $k_{z}$ and $\lambda_{z}$ are independent of $r$, but both $k_{\theta}$ and $\lambda_{\theta}$ are functions of $r$ (because $k_{\theta 0}$ is a function of $r$ ). Equation (10) can be alternatively expressed as a function of wavelengths as $\check{E}_{i i}\left(\lambda_{z}, \lambda_{\theta}, r\right)=2 \overline{\widehat{u}_{i}^{\prime}\left(\lambda_{z}, \lambda_{\theta}, r\right){\widehat{u_{i}^{\prime}}}^{*}\left(\lambda_{z}, \lambda_{\theta}, r\right)}$.

Figures $11(\mathrm{a})-11(\mathrm{~d})$ display contours of premultiplied $2 \mathrm{D}$ energy spectra $k_{z}^{+} k_{\theta}^{+} \check{E}_{z z}^{+}$of case B4 at two radial positions: close to the wall at $r^{+}=15$, where the axial component of TKE [i.e., $\left\langle u_{z}^{\prime} u_{z}^{\prime}\right\rangle$ ] peaks, and one quarter cylinder gap (or $\delta / 2)$ away from the wall [i.e., $(R-r) / \delta=0.5$ from the convex wall or $(r-R) / \delta=0.5$ from the concave wall]. The flow structures at these two radial positions are qualitatively different in the sense that streaky structures are populated in the near-wall region around $r^{+}=15$, while hairpin packets are populated around the elevated position $\delta / 2$ away from the convex or concave wall. In presenting results, the $2 \mathrm{D}$ energy spectrum, wavenumbers, and wavelengths have been non-dimensionalized using $v$ and local wall friction velocity (which equals either $u_{\tau i}$ or $u_{\tau o}$ depending on the convex or concave side). These non-dimensionalized quantities are indicated using superscript " + " in consistency with the convention of wall coordinates. In Fig. 11, the spectrum maps show three regions of high-, intermediate-, and low-intensity cores distinguished by colors, and their borders are shown using black solid lines corresponding to $0.875 \max \left(k_{z}^{+} k_{\theta}^{+} \breve{E}_{z z}^{+}\right), 0.625 \max \left(k_{z}^{+} k_{\theta}^{+} \check{E}_{z z}^{+}\right)$, and $0.375 \max \left(k_{z}^{+} k_{\theta}^{+} \check{E}_{z z}^{+}\right)$(or $7 / 8$ th, $5 / 8$ th, and $3 / 8$ th the peak values of the non-dimensionalized premultiplied spectrum, respectively). The high-intensity core enclosed by the innermost isopleth of $0.875 \max \left(k_{z}^{+} k_{\theta}^{+} \breve{E}_{z z}^{+}\right)$corresponds to the most energetic eddies of the turbulent flow field. Although the low-intensity core [enclosed by the outermost isopleth of $0.375 \max \left(k_{z}^{+} k_{\theta}^{+} \breve{E}_{z z}^{+}\right)$] corresponds to less dominant energetic eddies encompassing a large range length scales, it still contributes considerably to the total TKE of the flow.

Besides the three isopleths that show three magnitude levels of the premultiplied spectrum relative to its peak value, the absolute value of the non-dimensionalized premultiplied spectra is also indicated using a color legend in Fig. 11. The computational domain sizes of case B4 (i.e., $L_{z}=12 \pi \delta$ and $L_{\theta}=3 \pi / 4$ ) are shown as the boundaries in Fig. 11. For the purpose of comparison,

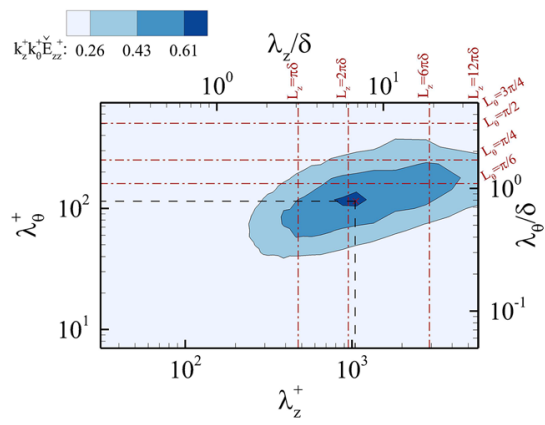

(a)

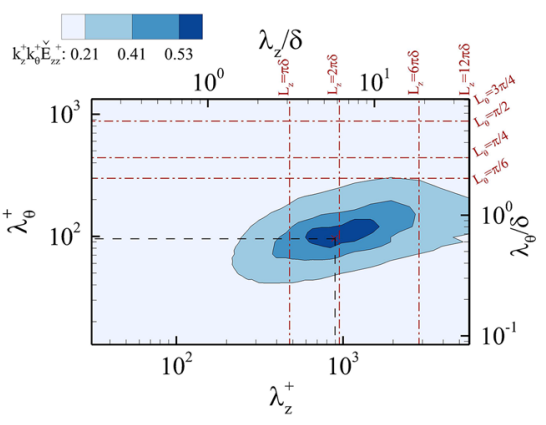

(b)

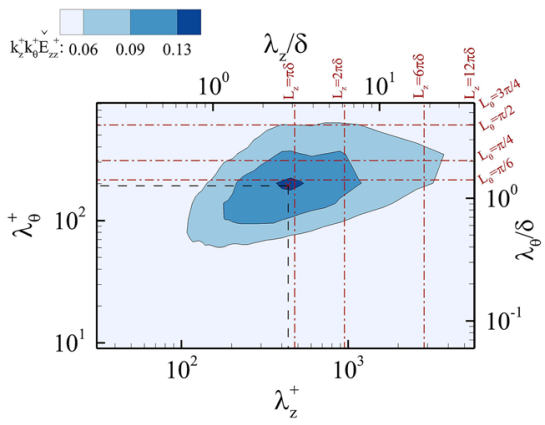

(c)

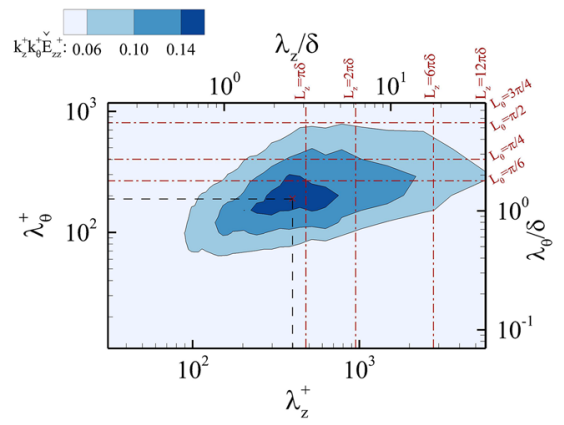

(d)

FIG. 11. Premultiplied 2D spectra $k_{z}^{+} k_{\theta}^{+} \check{E}_{z z}^{+}$of axial velocity fluctuations on the convex and concave sides of the concentric annular pipe for case B4, which vary with the axial and azimuthal wavelengths: (a) $r^{+}=15$ on the convex side, (b) $r^{+}=15$ on the concave side, (c) $(R-r) / \delta=0.5$ on the convex side, and (d) $(R-r) / \delta=0.5$ on the concave side. In each figure panel, the wavelength is given both as $\lambda_{z}^{+}$(bottom) and as $\lambda_{z} / \delta$ (top). Similarly, the azimuthal coordinate is given both as $\lambda_{\theta}^{+}$(left) and as $\lambda_{\theta} / \delta$ (right). The computational domain of case B4 (i.e., $L_{z}=12 \pi \delta$ and $L_{\theta}=3 \pi / 4$ ) is shown as the boundaries in Fig. 11. For the purpose of comparison, smaller axial and azimuthal domain sizes used in other test cases are also labeled in the figure. The figure presentations are made at two radial positions: close to the wall at $r^{+}=15$, where $\left\langle u_{z}^{\prime} u_{z}^{\prime}\right\rangle^{+}$ peaks, and at one quarter the cylinder gap (or $\delta / 2)$ away from the wall [i.e., $(R-r) / 2=0.5$ from the convex wall or $(r-R) / 2=0.5$ from the concave wall]. Three energy levels are distinguished, and the innermost, intermediate, and outermost isopleths correspond to $0.875 \max \left(k_{z}^{+} k_{\theta}^{+} \check{E}_{z z}^{+}\right), 0.625 \max \left(k_{z}^{+} k_{\theta}^{+} \check{E}_{z z}^{+}\right)$, and $0.375 \max \left(k_{z}^{+} k_{\theta}^{+} \check{E}_{z z}^{+}\right)$, respectively. 
smaller axial and azimuthal domain sizes used in other test cases are also labeled. From Fig. 11, it is clear that both the innermost and intermediate isopleths [corresponding to $0.875 \max \left(k_{z}^{+} k_{\theta}^{+} \check{E}_{z z}^{+}\right)$ and $0.625 \max \left(k_{z}^{+} k_{\theta}^{+} \breve{E}_{z z}^{+}\right)$, respectively] are fully captured. Furthermore, it is seen that the majority of the outermost isopleth of $0.375 \max \left(k_{z}^{+} k_{\theta}^{+} \check{E}_{z z}^{+}\right)$is captured, with only a very small portion of the isopleth missing in Figs. 11(a) and 11(b). Based on this analysis of premultiplied 2D spectra $k_{z}^{+} k_{\theta}^{+} \breve{E}_{z z}^{+}$, it is confirmed that the computational domain of case B4 (with $L_{z}=12 \pi \delta$ and $L_{z}=3 \pi / 4$ ) is satisfactory in general, which can facilitate capture of large flow structures up to wavelengths that correspond to at least $37.5 \%$ of the peak value of the non-dimensionalized premultiplied 2D spectrum. By contrast, any arbitrary combination of smaller axial and azimuthal computational domain sizes would result in an inaccurate prediction of largescale structures with a higher percentage of TKE cutoff. For instance, as shown in Fig. 11(c), if the "worst" combination of $L_{z}=\pi \delta$ and $L_{z}$ $=\pi / 6$ is used for conducting DNS, the domain is barely sufficient for capturing the peak of $k_{z}^{+} k_{\theta}^{+} \dot{E}_{z z}^{+}$but does not even allow for fully capturing the most energetic eddies of turbulence corresponding to the innermost isopleth of $0.875 \max \left(k_{z}^{+} k_{\theta}^{+} \check{E}_{z z}^{+}\right)$. The analysis conducted here is based on the premultiplied 2D spectra $k_{z}^{+} k_{\theta}^{+} \breve{E}_{z z}^{+}$at two special wall-normal positions of $r^{+}=15$ and $(r-R) / \delta=0.5$. Later in Secs. IV B and IV C, we will refine our study by further investigating the characteristic axial and azimuthal length scales of the most energetic turbulence structures over the entire wall-normal direction (i.e., the entire $r$-direction) through an analysis of their premultiplied 1D spectra.

Figures 11(a) and 11(b) show that the mode of $k_{z}^{+} k_{\theta}^{+} \check{E}_{z z}^{+}$occurs at $\lambda_{z}^{+} \approx 1100$ and $\lambda_{\theta}^{+} \approx 120$ on the convex side and at $\lambda_{z}^{+} \approx 900$ and $\lambda_{\theta}^{+}=90$ on the concave side. These two modes represent the characteristic length scales (periods) of the streaky structures in the axial and azimuthal directions. The differences in the axial and azimuthal characteristic wavelengths on the convex and concave sides result from the surface curvature difference between the convex and concave cylinder walls. Apparently, both axial and azimuthal characteristic length scales of the streaks are larger on the convex side than on the concave side. This leads to an important conclusion that the challenge involved in accurately performing DNS of a concentric annular pipe flow mostly stems from the need of capturing large streaky structures on the convex side. Compared to the concave side, the streaky structures on the convex side are more elongated in the axial direction and more widely spread in the azimuthal direction. Although the characteristic length scales of near-wall streaky structures in a concentric annular pipe flow are sensitive to the surface curvature, their magnitudes are, in general, comparable to those of a plane-channel flow, which are approximately 1000 and 100 wall units in the streamwise and spanwise directions, respectively. ${ }^{17,20}$

At a higher elevation of $\delta / 2$ away from the convex and concave walls, the curvature effect on turbulence structures reduces, and the flow is dominated by hairpin packets. From Figs. 11(c) and 11(d), it is seen that the mode of $k_{z}^{+} k_{\theta}^{+} \check{E}_{z z}^{+}$occurs approximately at $\lambda_{z}^{+}=405$ and $\lambda_{\theta}^{+}=190$ on both convex and concave sides. As is well known from the study of near-wall turbulent boundary layer over flat plates by Adrian, ${ }^{18}$ hairpin structures constantly generate secondary hairpin vortices (SHVs), develop into primary hairpin vortices (PHVs), and are destroyed as downstream hairpin vortices (DHVs). Figure 12 shows instantaneous contours of the swirling strength (for $\lambda_{c i}=1.0$ ) of case B4. Similar to Fig. 10, only part of the computational domain is used for illustrating hairpin structures in Fig. 12. In addition, the domain is divided radially into two parts to clearly show the hairpin structures on the convex and concave sides. The hairpin structures identified by the swirling strength are further superimposed with colors corresponding to the value of non-dimensionalized instantaneous axial vorticity $\omega_{z}^{\prime+}$, with red and blue colors representing its positive and negative values, respectively. This facilitates identification of paired hairpin legs of counter-rotating directions. Figure 12 shows that hairpin structures are populated on both convex and concave sides of the concentric annular pipe, consisting of elongated legs (appearing as counter-rotating vortices, differentiated using the blue and red colors of $\omega_{z}^{\prime+}$ ) near the wall and arches (or heads) at higher elevations relatively far away from the wall.

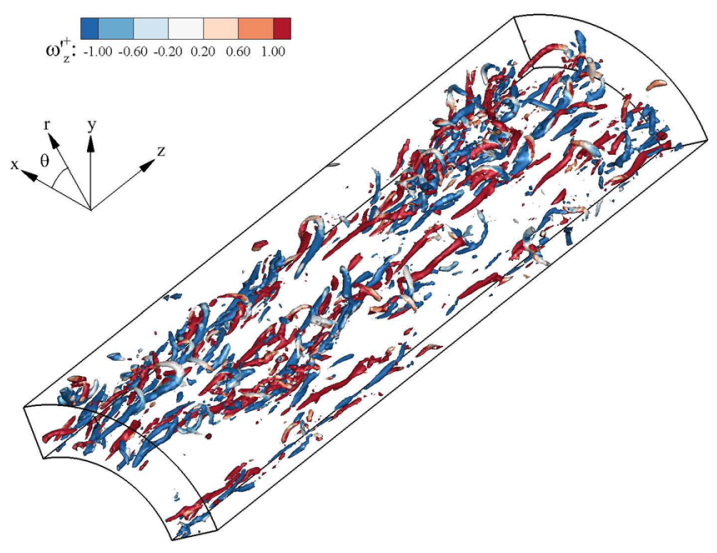

(a)

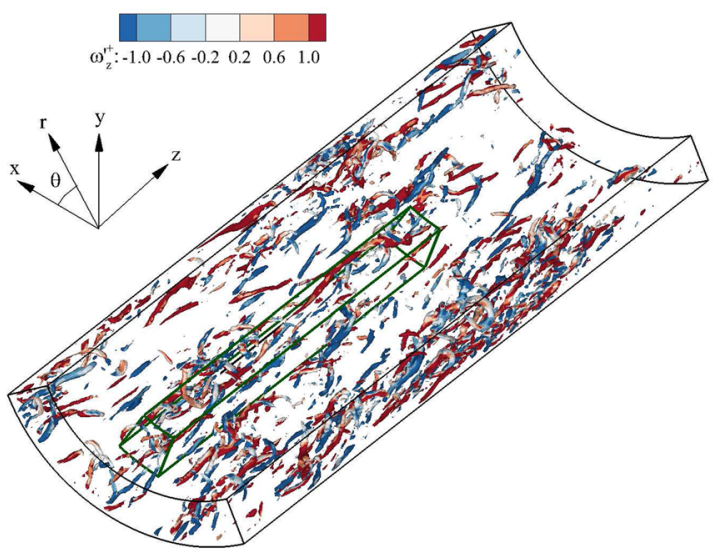

(b)

FIG. 12. Contours of swirling strength $\left(\lambda_{c i}=1.0\right)$ for case B4: (a) convex side and (b) concave side. The contours are colored with instantaneous axial vorticity $\omega_{z}^{\prime+}$. To enhance the visual clarity of near-wall structures, only a portion of the computational domain is plotted for $L_{z}=6 \pi \delta$ and $L_{\theta}=\pi / 2$. 
In order to demonstrate the spatial evolution of hairpin packets in the axial direction, a chain of hairpin structures on the concave side of the pipe are isolated from the surrounding flow structures in Fig. 12(b) using a green box and are shown separately by projecting the selected domain into a 2D $z-r$ plane in Fig. 13(a). The hairpin structures displayed in Fig. 13(a) are magnified, showing a clear side view of the axial development of hairpin packets (consisting of PHV and SHV) on the concave side of the concentric pipe. The hairpin structures can also be studied based on a conditional averaging method described by Adrian. ${ }^{21}$ Figure 13(b) shows the contours of $\left\langle u_{z}^{\prime+} \mid Q 2\right\rangle$, where the averaging of axial velocity fluctuations $u_{z}^{\prime}$ is done based on the condition of ejection events (or the Q2 events that feature $u^{\prime}<0$ and $v^{\prime}>0$ ). The reason that $u_{z}^{\prime}$ is selected is that the length scales of large-scale streaky structures (or the "legs" of hairpin structures) can be very effectively determined by the axial velocity fluctuations in either two-point coefficient analysis or 1D and 2D spectral analyses (see Figs. 9-11, 14, and 15). From both Figs. 13(a) and 13(b), the periodicity of the hairpin packets can be readily identified (indicated using the thick dashed lines), with a period of approximately $\lambda_{z}^{+}=400$. This result is consistent with our previous analysis of the mode of premultiplied $2 \mathrm{D}$ spectrum $k_{z}^{+} k_{\theta}^{+} \check{E}_{z z}^{+}$based on Fig. 11, which indicates that the axial characteristic wavelength of hairpin structures is approximately $\lambda_{z}^{+}=405$.

The above analysis of large-scale turbulence structures (specifically, near-wall streaks and hairpin structures in core turbulent regions) was conducted based on premultiplied 2D spectra $k_{z}^{+} k_{\theta}^{+} \check{E}_{z z}^{+}\left(r^{+}, \lambda_{z}^{+}, \lambda_{\theta}^{+}\right)$at two radial positions only. In order to develop a general understanding of the effect of radial position on the axial and azimuthal characteristic length scales of turbulence structures, in the following, we will refine the research by looking into the premultiplied 1D spectra as a function of $r^{+}$and $k_{z}^{+}$in Sec. IV B and as a function of $r^{+}$and $k_{\theta}^{+}$in Sec. IV C. In addition, through the study of characteristic length scales of turbulence structures using the premultiplied 1D spectra, we will further investigate the minimal axial domain size required for rigorously conducting DNS of concentric annular pipe flow and structures.

\section{B. Axial premultiplied energy spectra}

The $1 \mathrm{D}$ axial energy spectrum can be directly computed by integrating the $2 \mathrm{D}$ energy spectrum over the azimuthal wavenumbers, i.e.,

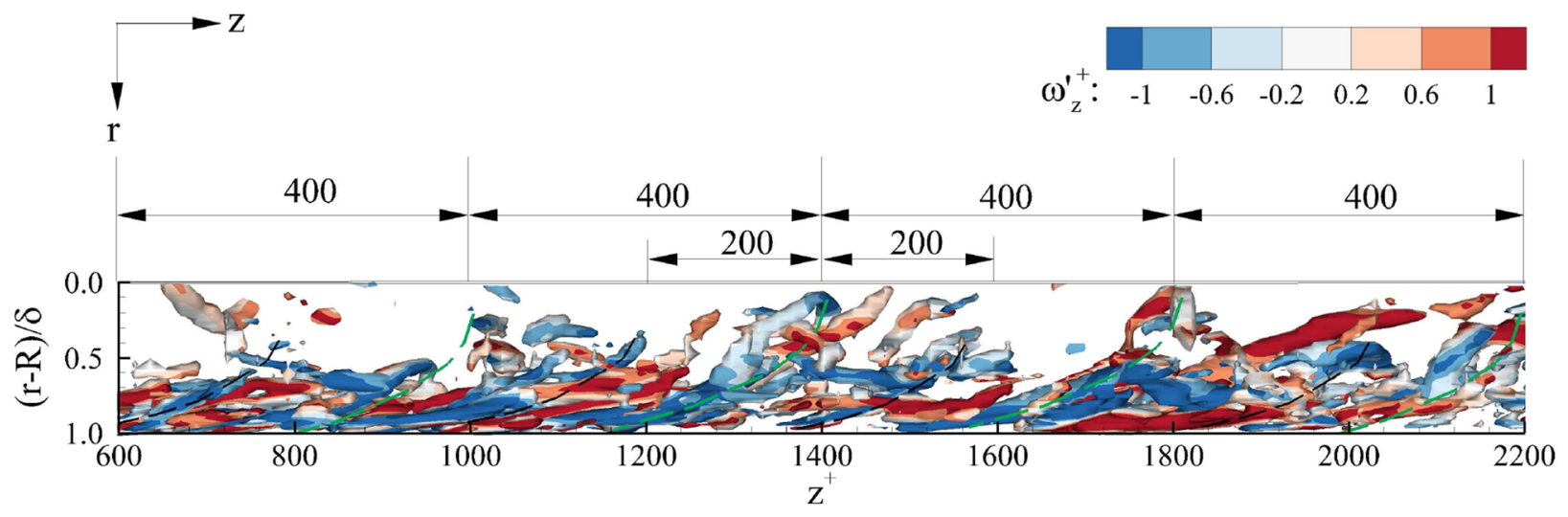

(a)

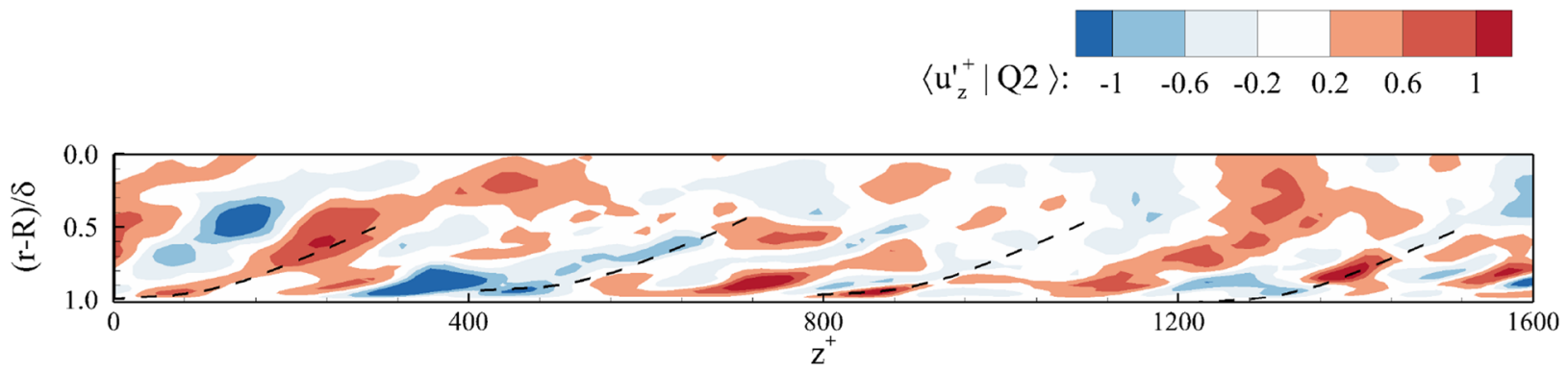

(b)

FIG. 13. Side view of hairpin structures on the concave side of the concentric pipe for case B4. (a) Contours of the swirling strength of $\lambda_{c i}=1.0$ colored using the strength of $\omega_{z}^{\prime+}$ and (b) contours of conditional averaging of axial velocity fluctuations $\left\langle u_{z}^{\prime+} \mid Q 2\right\rangle$ in the $z-r$ plane located at $\theta=0^{\circ}$. In panel (a), the hairpin structures are obtained by projecting the selected domain from Fig. 12(b) (indicated using a 3D green box) into a 2D z-r plane here. The green and black dashed lines in panel (a) show the positions of the PHV and SHV, respectively. The wavelength of both PHV and SHV is approximately $\lambda_{z}^{+}=400$. 


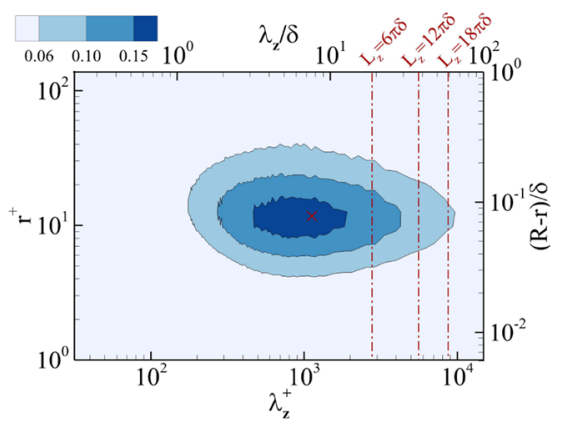

(a)

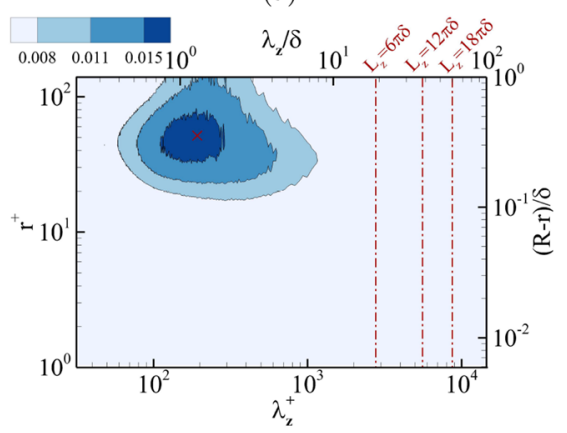

(c)

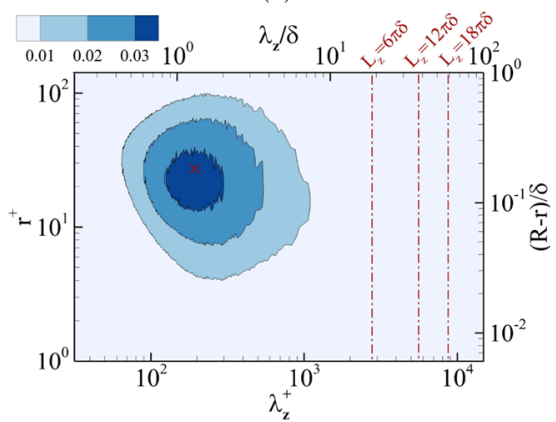

(e)

$$
\widetilde{E}_{i i}\left(k_{z}, r\right)=\int \check{E}_{i i}\left(k_{z}, k_{\theta}, r\right) d k_{\theta},
$$

or in a discrete form, as $\widetilde{E}_{i i}\left(k_{z}, r\right)=k_{\theta 0} \sum_{k_{\theta}} \check{E}_{i i}\left(k_{z}, k_{\theta}, r\right)$ for $i=1$, 2 , or 3 (no summation convention implied). Due to the need of studying cases of different domain sizes (of different values of $k_{z 0}$ and $k_{\theta 0}$ ), the premultiplied $1 \mathrm{D}$ energy spectrum $k_{z} \widetilde{E}_{i i}$ needs to be further normalized by the spectral area $k_{z 0} k_{\theta 0}$ in our comparative study. Because case A6 has the largest computational domain size, its spectral results are the most accurate among all 12 test cases of the lower nominal Reynolds number of $R e_{D_{h}}=8900$. Figure 14 compares the premultiplied 1D axial energy spectra of all three velocity fluctuation components on the convex and concave sides of case A6. For the purpose of comparison, vertical dashed-dotted lines are used to demarcate the axial computational domain sizes of cases A3-A5, which vary from $L_{z}=6 \pi \delta$ to $18 \pi \delta$. The premultiplied 1D energy spectrum has been non-dimensionalized using the local friction velocity, which equals either $u_{\tau i}$ or $u_{\tau o}$ depending on the convex or the concave cylinder side in consideration. Similar to Fig. 11, three levels of the premultiplied energy spectrum are identified, which represent the high-, intermediate-, and lowintensity cores of a turbulence structure. The contours for these three levels of the premultiplied spectrum are distinguished by colors and black solid borderlines corresponding to $0.875 \max \left(k_{z}^{+} \widetilde{E}_{i i}^{+}\right)$, $0.625 \max \left(k_{z}^{+} \widetilde{E}_{i i}^{+}\right)$, and $0.375 \max \left(k_{z}^{+} \widetilde{E}_{i i}^{+}\right)$.

Figures 14(a) and 14(b) compare the contour patterns of the non-dimensionalized premultiplied 1D spectrum of axial velocity fluctuations $k_{z}^{+} \widetilde{E}_{z z}^{+}$on the convex and concave sides of the concentric annular pipe, as a function of the radius $r^{+}$and wavelength $\lambda_{z}^{+}$. The mode (indicated using a red cross symbol " $x$ " in the figure) of $k_{z}^{+} \widetilde{E}_{z z}^{+}$occurs at $\left(r^{+}, \lambda_{z}^{+}\right)=(14.8,1100)$ and $\left(r^{+}, \lambda_{z}^{+}\right)=(15.3,900)$ on the convex and concave sides, respectively. Clearly, the characteristic axial length scales of the streaky structures as indicated by the axial modes of $k_{z}^{+} \widetilde{E}_{z z}^{+}$and $k_{\theta}^{+} k_{z}^{+} \breve{E}_{z z}$ (inferred from Figs. 11 and 14) are consistent, which are $\lambda_{z}^{+}=1100$ and 900 on the convex and concave 


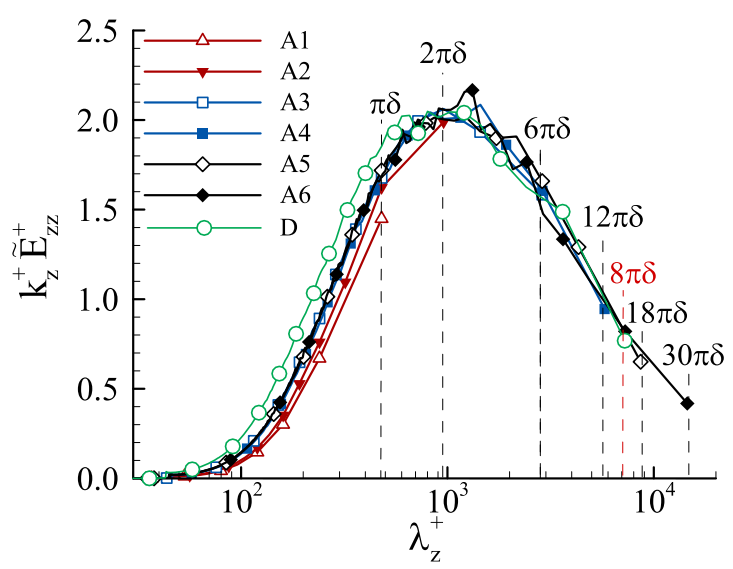

(a)

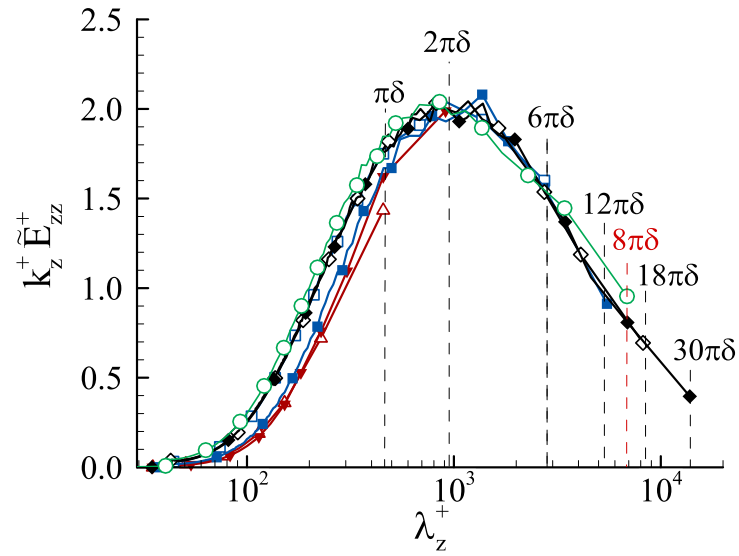

(b)

FIG. 15. Profiles of non-dimensionalized premultiplied $1 \mathrm{D}$ axial energy spectra $k_{z}^{+} \widetilde{E}_{z z}^{+}$of the A- and D-series test cases at the cylindrical surface located at $r^{+}=15$ from the (a) convex and (b) concave walls. Vertical dashed lines demarcate the axial computational sizes (i.e., $L_{z}$ values) for cases A1-A6 and D.

sides of the concentric annular pipe, respectively. The radial position where the mode of $k_{z}^{+} \widetilde{E}_{z z}^{+}$occurs is similar between the convex and concave sides of the concentric annular pipe, which is approximately at $r^{+}=15$. This implies that under the testing condition, the near-wall streaky structures are the most energetic at this radial position on both sides of the concentric annular pipe. The appearance of the peak value of $k_{z}^{+} \widetilde{E}_{z z}^{+}$at the radial position of approximately $r^{+}$ $=15$ is consistent with that for $\left\langle u_{z}^{\prime} u_{z}^{\prime}\right\rangle^{+}$in the physical space. However, it should be indicated here that the overlapping of these two modes of $k_{z}^{+} \widetilde{E}_{z z}^{+}$and $\left\langle u_{z}^{\prime} u_{z}^{\prime}\right\rangle^{+}$at $r^{+}=15$ is not strictly required in mathematics because these two quantities are connected through an integral relationship, i.e., $\left\langle u_{z}^{\prime} u_{z}^{\prime}\right\rangle=\int \widetilde{E}_{z z}\left(r, k_{z}\right) d k_{z}$. From Figs. 14(a) and 14(b), it is evident that the domain of case A6 (with $L_{z}=30 \pi \delta$ and $L_{\theta}=2 \pi$ ) is sufficiently large to fully capture the outermost isopleth that corresponds to $0.375 \max \left(k_{z}^{+} \widetilde{E}_{i i}^{+}\right)$. If the axial domain size drops to $L_{z}=18 \pi \delta$ (as in case A5), the outermost isopleth is still fully captured on the concave side of the pipe at all radial positions. As the axial domain size drops further to $L_{z}=12 \pi \delta$ (as in case A4), the outermost isopleth is well captured at all radial positions but is slightly missed around $r^{+}=15$. However, as is shown in Fig. 14(a), if the axial domain size continues to drop to $L_{z}$ $=6 \pi \delta$ (as in case A3), even the intermediate isopleth corresponding to a higher TKE level of $0.625 \max \left(k_{z}^{+} \widetilde{E}_{i i}^{+}\right)$cannot be fully captured, indicating a rather inaccurate DNS. From the previous analysis of turbulence statistics in the physical space conducted in Sec. III, we drew a conclusion that a minimum axial domain length of $L_{z}$ $=6 \pi \delta$ of case A 3 was satisfactory, which is apparently contradictory to the evidence shown in Figs. 14(a) and 14(b). The spectral analysis conducted here allows us to refine the study by accurately assessing the axial characteristic wavelengths of energetic near-wall streaky structures. Based on the combined physical and spectral analyses, it is now confirmed that the minimum axial computation domain must be stretched to $L_{z}=12 \pi \delta$, in order to perform DNS accurately for the turbulent concentric annular pipe flow investigated here.
By comparing Figs. 14(c)-14(f), it is apparent that all three isopleths [corresponding to $0.875 \max \left(k_{z}^{+} \widetilde{E}_{i i}^{+}\right), 0.625 \max \left(k_{z}^{+} \widetilde{E}_{i i}^{+}\right)$, and $\left.0.375 \max \left(k_{z}^{+} \widetilde{E}_{i i}^{+}\right)\right]$are fully captured by axial premultiplied 1D spectra of radial and azimuthal velocity fluctuations, $k_{z}^{+} \widetilde{E}_{r r}^{+}$and $k_{z}^{+} \widetilde{E}_{\theta \theta}^{+}$, respectively. It should be indicated that in Figs. 14(c) and 14(d), the outermost isopleth is not fully captured at the maximum radial position around $r^{+}=150$. This is not an indication of insufficient radial domain size but instead a reflection of the radial domain center, as only one-half of the radial domain is plotted in Figs. 14(c) and 14(d). From Figs. 14(c)-14(f), it is observed that the energy levels of $k_{z}^{+} \widetilde{E}_{r r}^{+}$ and $k_{z}^{+} \widetilde{E}_{\theta \theta}^{+}$(as indicated by the color-scale legend) are one order of magnitude smaller than that of $k_{z}^{+} \widetilde{E}_{z z}^{+}$. The modes of $k_{z}^{+} \widetilde{E}_{r r}^{+}$and $k_{z}^{+} \widetilde{E}_{\theta \theta}^{+}$ occur at $\left(r^{+}, \lambda_{z}^{+}\right)=(50,200)$ and $(30,200)$ on both sides of the concentric pipe, respectively. Clearly, the axial characteristic wavelengths of the turbulence structures as indicated by the modes of $k_{z}^{+} \widetilde{E}_{r r}^{+}$and $k_{z}^{+} \widetilde{E}_{\theta \theta}^{+}$are much smaller than that of $k_{z}^{+} \widetilde{E}_{z z}^{+}$(which is about 1000 wall units, see above). Furthermore, it is interesting to observe that the modes of $k_{z}^{+} \widetilde{E}_{z z}^{+}, k_{z}^{+} \widetilde{E}_{r r}^{+}$, and $k_{z}^{+} \widetilde{E}_{\theta \theta}^{+}$appear at three different radial positions for $r^{+}=15,30$, and 50 , respectively. The physical mechanisms underlying these observations relate to the features of hairpin structures shown in Fig. 13. Similar to the hairpin structures of a turbulent boundary layer developed over a flat plate, ${ }^{18}$ the radially oriented parts of a hairpin structure that connects the legs to the head (referred to as the "neck" by Adrian ${ }^{18}$ ) contribute the most to the peak value of $k_{z}^{+} \widetilde{E}_{r r}^{+}$(associated with the radial Reynolds normal stress component $\left\langle u_{r}^{\prime} u_{r}^{\prime}\right\rangle^{+}$). The necks of hairpin structures (for both PHV and SHV) are the most energetic around $r^{+}=50$, characterized by a wavelength of approximately $\lambda_{z}^{+}=200$. Because the characteristic wavelength of either PHV or SHV (see Fig. 13) is approximately 400 wall units, the axial separation of the hairpin necks is typically around 200 wall units at this particular radial position judging from the mode of $k_{z}^{+} \widetilde{E}_{r r}^{+}$. The reason that the peak of $k_{z}^{+} \widetilde{E}_{\theta \theta}^{+}$appears at $r^{+}=30$ is that there exist azimuthally oriented vortex filaments, which connect hairpin legs and are the most energetic around this elevation. These azimuthally oriented vortex filaments lay further 
away from the wall than the near-wall streaks, perturbed and pushed away by ejections to form hairpin arches at higher elevations. Consistent with the analysis of the hairpin necks, the characteristic wavelength that represents the axial separation of azimuthally oriented vortex filaments is also 200 wall units.

From the above analysis of the premultiplied $1 \mathrm{D}$ energy spectrum $k_{z}^{+} \widetilde{E}_{z z}^{+}$in Figs. 14(a) and 14(b), it is understood that the axially elongated streaks are the most energetic and the longest at $r^{+} \approx 15$ on both convex and concave sides. Correspondingly, the magnitude of Reynolds normal stress $\left\langle u_{z}^{\prime} u_{z}^{\prime}\right\rangle^{+}$also peaks at this radial position on both sides of the concentric annular pipe. The contours of $k_{z}^{+} \widetilde{E}_{z z}^{+}$ shown in Fig. 14 are based on case A6 only, which has the largest computational domain and allows for the most accurate prediction of turbulence statistics. However, in order to understand the direct influence of the axial domain size on the predictive accuracy of $k_{z}^{+} \widetilde{E}_{z z}^{+}$, test cases of different axial domain sizes must be compared based on independent numerical simulations. To this purpose, the profiles of all six A-series test cases are compared in Fig. 15. To demonstrate the Reynolds number effect, the profiles of the six A-series test cases are also compared against that of case D. The black dashed lines demarcate the axial computational domain sizes as well as the cutoff wavelengths of the A-series test cases, which vary from $L_{z}$ $=6 \pi \delta$ to $30 \pi \delta$ in cases A3-A6. Similarly, the red dashed line demarcates the axial computational domain size and cutoff wavelength of case D.

From Fig. 15, it is clear that cases A1 and A2 fail to capture the mode of $k_{z}^{+} \widetilde{E}_{z z}^{+}$, implying that the most energetic eddy motions are missed in DNS. This well explains the failure of cases A1 and A2 in terms of the prediction of $\left\langle u_{z}^{\prime} u_{z}^{\prime}\right\rangle^{+}$in Fig. 4(a). As is clear from Fig. 15, although the mode of the premultiplied 1D energy spectrum has been successfully captured in cases A3-A6, the spectrum cutoffs for these four test cases are different due to the differences in their axial domain sizes. At their cutoff wavelength, the magnitude of the premultiplied $1 \mathrm{D}$ energy spectrum is $79 \%, 45 \%, 32 \%$, and $21 \%$ of its peak value on the convex side and is $79 \%, 44 \%, 34 \%$, and $18 \%$ of its peak value on the concave side in cases A3, A4, A5, and A6, respectively. Given the fact that the cutoff wavelength occurs at a very high TKE level, the amount of TKE associated with wavelengths that are larger than the cutoff wavelength missed by DNS is significant in case A3. For this reason, even though the axial domain length of case A3 $\left(L_{z}=6 \pi \delta\right)$ was satisfactory in previous predictions of the mean velocity, Reynolds stresses, and two-point correlation coefficient, it is insufficient with respect to the current spectral analysis. Based on our previous analysis of Fig. 14, it is understood that an axial domain size of $L_{z}=12 \pi \delta$ is satisfactory in general with respect to capturing TKE at all radial positions for test cases of a lower nominal Reynolds number of $R e_{D_{h}}=8900$. From Fig. 15, it is further confirmed that even at $r^{+}=15$ where the near-wall streaks are the most energetic and the longest, the cutoff wavelength occurs when the premultiplied $1 \mathrm{D}$ spectrum decays to $45 \%$ of its peak value, implying that most of the energy-containing length scales are resolved in DNS.

From Fig. 15, it is seen that as the nominal Reynolds number increases from $R e_{D_{h}}=8900$ to 17700 , the cutoff wavelength of case D takes place when the premultiplied $1 \mathrm{D}$ spectrum decays to $39 \%$ and $47 \%$ of its peak value on the convex and concave cylinder sides, respectively. By comparing case D with case A4, it is clear that although the level of the premultiplied $1 \mathrm{D}$ spectrum at the cutoff wavelength is comparable between cases D and A4, the axial domain length of case $\mathrm{D}$ is only $L_{z}=8 \pi \delta$, which is much shorter than that $(12 \pi \delta)$ of case A4. This implies that a shorter axial domain is needed for conducting DNS at a higher Reynolds number. This phenomenon is interesting but not surprising, which is similar to that of the classical turbulent plane-channel flows ${ }^{11,17,19}$ and can be explained as follows. From the above analysis of Figs. 14 and 15, it understood that the mode of either the $2 \mathrm{D}$ or $1 \mathrm{D}$ premultiplied spectrum of axial velocity fluctuations occurs approximately at a wavelength of $\lambda_{z}^{+}=u_{\tau} \lambda_{z} / v \approx 1000$ at both nominal Reynolds numbers, which corresponds to the characteristic axial length scale of the most energetic streaky structures. Considering that the value of wall friction velocity $u_{\tau}$ increases as the Reynolds number increases (see Table II), then the characteristic wavelength $\lambda_{z}$ (corresponding to the mode) must decrease in order to maintain the value of the non-dimensional wavelength $\lambda_{z}^{+}$at approximately 1000 wall units. As such, the physical axial length of the concentric annular pipe required for capturing the most energetic streaky structures can be shortened as the Reynolds number increases.

For the lower nominal Reynolds number tested $\left(R e_{D_{h}}=8900\right)$, although the axial computational domain size of case A6 is the largest, eddies of axial length scales that are larger than the cutoff wavelengths (where the value of $k_{z}^{+} \widetilde{E}_{z z}^{+}$decays to $21 \%$ and $18 \%$ of its peak value on the convex and concave sides, respectively) are still missed in the DNS. In fact, it is unrealistic to fully capture the premultiplied energy spectrum (with $0 \%$ of spectrum leaking) in a DNS or in a physical experiment as it demands using a pipe of infinite length. Thus far, the longest axial domain size used for DNS of concentric annular pipe flow in the literature is $L_{z}=6 \pi \delta$ by Chung et $a l .{ }^{8}$ for a similar nominal Reynolds number of $R e_{D_{h}}=8900$. As such, the minimum axial domain size of $L_{z}=12 \pi \delta$ recommended here is twice that of Chung et al., which makes it the longest in the current literature.

\section{Azimuthal premultiplied energy spectra}

Similar to the 1D axial energy spectrum, the 1D azimuthal energy spectrum can be computed by integrating the $2 \mathrm{D}$ energy spectrum over the axial wavenumbers as

$$
\widetilde{E}_{i i}\left(k_{\theta}, r\right)=\int \check{E}_{i i}\left(k_{z}, k_{\theta}, r\right) d k_{z}
$$

or in a discrete form as $\widetilde{E}_{i i}\left(k_{\theta}, r\right)=k_{z 0} \sum_{k_{z}} \check{E}_{i i}\left(k_{z}, k_{\theta}, r\right)$ for $i=1$, 2 , or 3 (no summation convention implied). Different from the $1 \mathrm{D}$ axial energy spectrum, the 1D azimuthal energy spectrum is useful for analyzing the characteristic azimuthal length scales associated with the energetic eddy motions. Based on the conclusion of Subsection IV B, it is understood that a minimum axial domain of $L_{z}=12 \pi \delta$ is needed in order to capture axially elongated energetic eddy motions. In this subsection, we aim at investigating the minimum azimuthal domain size for DNS by analyzing the premultiplied 1D azimuthal energy spectrum $k_{\theta}^{+} \widetilde{E}_{i i}^{+}$(as a function of $r^{+}$and $k_{\theta}^{+}$). To this purpose, case B5 (with $L_{z}=12 \pi \delta$ and $L_{\theta}=2 \pi$ ) is selected, which has a sufficient axial domain size and the largest azimuthal domain size to facilitate generation of the most reliable azimuthal spectral results. Figure 16 compares the premultiplied 1D azimuthal energy spectra of all three velocity fluctuation components on the convex and concave sides of case B5. Dashed-dotted lines demarcate the 


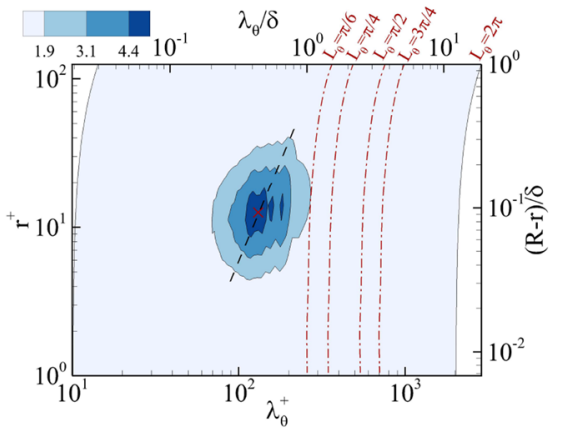

(a)

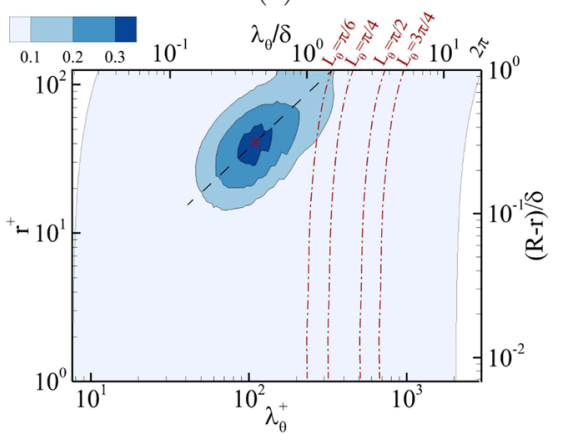

(c)

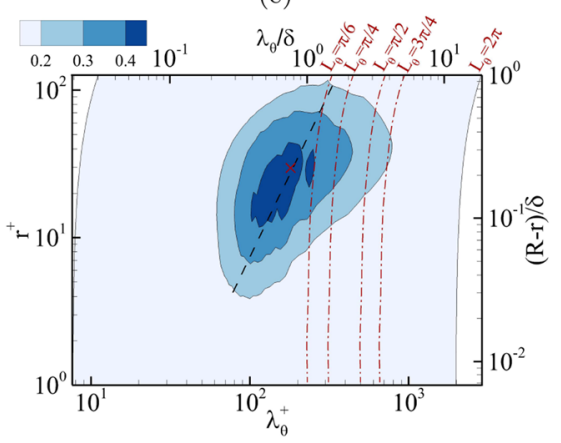

(e)

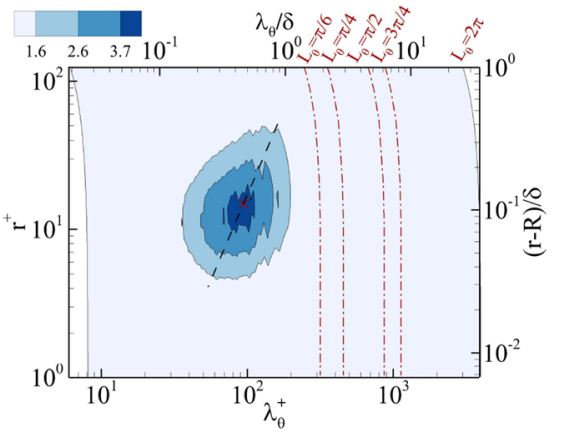

(b)

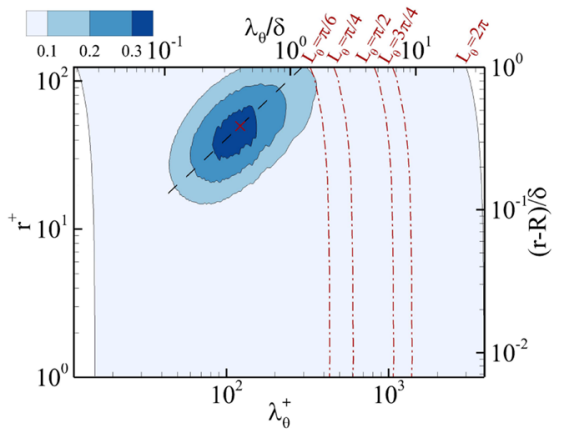

(d)

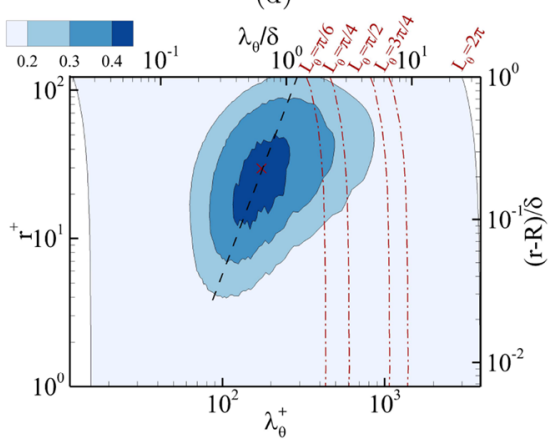

(f)
FIG. 16. Contours of premultiplied 1D axial energy spectra of $k_{\theta}^{+} \widetilde{E}_{z z}^{+}\left(\lambda_{z}^{+}, r^{+}\right)$, $k_{\theta}^{+} \widetilde{E}_{r r}^{+}\left(\lambda_{z}^{+}, r^{+}\right)$, and $k_{\theta}^{+} \widetilde{E}_{\theta \theta}^{+}\left(\lambda_{z}^{+}, r^{+}\right)$of case $\mathrm{B} 5$, non-dimensionalized by $u_{\tau}^{2}$. (a) $k_{\theta}^{+} \widetilde{E}_{z z}^{+}$on the convex side, (b) $k_{\theta}^{+} \widetilde{E}_{z z}^{+}$ on the concave side, (c) $k_{\theta}^{+} \widetilde{E}_{r r}^{+}$on the convex side, (d) $k_{\theta}^{+} \widetilde{E}_{r r}^{+}$on the concave side, (e) $k_{\theta}^{+} \widetilde{E}_{\theta \theta}^{+}$on the convex side, and (f) $k_{\theta}^{+} \widetilde{E}_{\theta \theta}^{+}$on the concave side. The value of $u_{\tau}$ equals either $u_{\tau i}$ or $u_{\tau 0}$, depending on the convex or the concave cylinder side in consideration. In each figure panel, the wavelength is given both as $\lambda_{\theta}^{+}$(bottom) and as $\lambda_{\theta} / \delta$ (top). Similarly, the radial coordinate is given both as $r^{+}$(left) and as $(R-r) / \delta$ or $(r-R) / \delta$ (right, for the convex or concave side, respectively). The cross symbol " $x$ " pinpoints the location of the mode. Three energy levels are distinguished, and the innermost, intermediate, and outermost isopleths correspond to $\phi_{i i}$ $=0.875 \max \left(k_{\theta}^{+} \widetilde{E}_{i i}^{+}\right), 0.625 \max \left(k_{\theta}^{+} \widetilde{E}_{i i}^{+}\right)$, and $0.375 \max \left(k_{\theta}^{+} \widetilde{E}_{i i}^{+}\right)$, respectively. Besides these three isopleths that show three magnitude levels of the premultiplied spectrum relative to its peak value, the absolute magnitude of the non-dimensionalized premultiplied spectrum is also shown using a color band. Vertical dashed-dotted lines demarcate the axial computational sizes for cases B1-B5. azimuthal computational domain sizes of cases B1-B5, which vary from $L_{\theta}=\pi / 6$ to $L_{\theta}=2 \pi$. Similar to Subsection IV B, the local friction velocity $\left(u_{\tau i}\right.$ or $\left.u_{\tau o}\right)$ is used for non-dimensionalization on the convex or concave side. The cross symbol " $x$ " pinpoints the mode corresponding to the maximum premultiplied energy spectrum, i.e., $\max \left(k_{\theta}^{+} \widetilde{E}_{i i}^{+}\right)$. Similar to the previous analysis, contours corresponding to the of high-, intermediate-, and low-intensity cores are distinguished by three isopleth values of the premultiplied 1D azimuthal spectra of $0.875 \max \left(k_{\theta}^{+} \widetilde{E}_{i i}^{+}\right), 0.625 \max \left(k_{\theta}^{+} \widetilde{E}_{i i}^{+}\right)$, and $0.375 \max \left(k_{\theta}^{+} \widetilde{E}_{i i}^{+}\right)$, respectively. By comparing Figs. 16(a)-16(f), it is clear that Fig. 16(e) represents the most critical scenario in terms of the choice of the azimuthal domain size, which shows that a minimal domain of $L_{\theta}=3 \pi / 4$ (corresponding to case B4) is needed in order to fully capture the outermost isopleth corresponding to $0.375 \max \left(k_{\theta}^{+} \tilde{E}_{\theta \theta}^{+}\right)$.
Figures 16(a) and 16(b) compare the contour patterns of the premultiplied 1D azimuthal spectrum of axial velocity fluctuations $\left(k_{\theta}^{+} \widetilde{E}_{z z}^{+}\right)$on the convex and concave sides. The mode of $k_{\theta}^{+} \widetilde{E}_{z z}^{+}$occurs at $\left(r^{+}, \lambda_{\theta}^{+}\right)=(14.8,120)$ and $\left(r^{+}, \lambda_{\theta}^{+}\right)=(15.3,90)$ on the convex and concave sides, respectively. The small difference in the modal values of $r^{+}$between the convex and concave sides is due to the surface curvature effect. Nonetheless, the appearance of the peak value of $k_{\theta}^{+} \widetilde{E}_{z z}^{+}$ at the radial position of approximately $r^{+}=15$ is consistent with the previous conclusion based on the analysis of the premultiplied 1D axial spectrum $k_{z}^{+} \tilde{E}_{z z}^{+}$.

Figures 16(c) and 16(d) show contours of the premultiplied $1 \mathrm{D}$ azimuthal spectrum of radial velocity fluctuations $k_{\theta}^{+} \widetilde{E}_{r r}^{+}$on the convex and concave cylinder sides, respectively. Clearly, the mode occurs at $\left(r^{+}, \lambda_{\theta}^{+}\right) \approx(50,100)$ on both cylinder sides of the concentric annular pipe. This mode of $k_{\theta}^{+} \tilde{E}_{r r}^{+}$indicates that the radially 
oriented parts of hairpin structures (i.e., hairpin necks) are the most energetic at the radial position of $r^{+} \approx 15$ and the azimuthal spacing is approximately 100 wall units. Figures 16(e) and 16(f) show the contours of the premultiplied 1D azimuthal spectrum of azimuthal velocity fluctuations $k_{\theta}^{+} \widetilde{E}_{\theta \theta}^{+}$. From Figs. 16(e) and 16(f), it is seen that the mode of $k_{\theta}^{+} \widetilde{E}_{\theta \theta}^{+}$occurs at $\left(r^{+}, \lambda_{\theta}^{+}\right) \approx(30,150)$ on both convex and concave sides of the concentric annular pipe. The physical mechanism underlying this mode is that the vortex filaments that connect the legs of the hairpin structures (i.e., hairpin arches) are the most energetic at $r^{+} \approx 30$, with an characteristic azimuthal wavelength of $\lambda_{\theta}^{+}=150$. It should be further indicated here that although the hairpin necks are the most energetic at $r^{+} \approx 50$, this does not imply that the hairpin arches are necessarily the most energetic at higher elevations for $r^{+}>50$. This is because the structures and dynamics of hairpin packets are always more complicated in reality than the ideal situation that all hairpin structures are uniformly sized and each hairpin structure has a perfect symmetrical shape of two legs, two necks, and one arch.

In Figs. 16(a)-16(e), the contours of all three components of $k_{\theta}^{+} \widetilde{E}_{i i}^{+}$show quasi-elliptical shapes, with an inclined major axis. This inclined major axis shows a linear scale growth rate with the wall-normal distance, which indicates that the azimuthal scales of near-wall streaks [Figs. 16(a) and 16(b)] and hairpin structures [Figs. 16(c)-16(f)] increase as the wall-normal distance increases. This observation is consistent with the DNS results of turbulent pipe flows of Wu et al. ${ }^{22}$ and hot-wire measurements of turbulent channel and pipe flows of Monty et al.

From the above analysis of Fig. 16, it is understood that the value of $k_{\theta}^{+} \widetilde{E}_{\theta \theta}^{+}$is more sensitive to the choice of the azimuthal domain size than the other two components of the premultiplied $1 \mathrm{D}$ azimuthal spectra. Furthermore, it is understood that the analysis of Fig. 16 is conducted based on the DNS result of case B5 only, which has the largest azimuthal domain size of $L_{\theta}=2 \pi$ and offers the most accurate DNS results among the B-series test cases. However, in order to examine the actual influence of the azimuthal domain size on the predictive accuracy of DNS, numerical simulations based on all five B-series test cases need to be carried independently. To this purpose, Fig. 17 compares the non-dimensionalized premultiplied 1D azimuthal spectra $k_{\theta}^{+} \tilde{E}_{\theta \theta}^{+}$of all five B-series test cases at a fixed radial position $r^{+}=30$. The reason that Fig. 17 is plotted for $r^{+}=30$ is that $k_{\theta}^{+} \widetilde{E}_{\theta \theta}^{+}$peaks approximately at this radial position based on the DNS result of case B5 shown in Figs. 16(e) and 16(f). In Fig. 17, the black dashed lines demarcate the azimuthal computational domain sizes as well as the cutoff wavelengths of the B-series test cases, which vary from $L_{\theta}=\pi / 6$ to $2 \pi$ in cases B1 to B5. The results shown in Fig. 17 provide a direct measure on the accuracy of the assumed periodical azimuthal boundary condition used in DNS at the lower nominal Reynolds number of $R e_{D_{h}}=8900$. To investigate the Reynolds number effects, the profiles of these five B-series test cases are also compared against that of case D. Clearly, the turbulence energy level as indicated by the premultiplied spectrum of the azimuthal velocity fluctuations $\left(k_{\theta}^{+} \tilde{E}_{\theta \theta}^{+}\right)$increases as the Reynolds number increases. This pattern of $k_{\theta}^{+} \tilde{E}_{\theta \theta}^{+}$as a result of an increase in the Reynolds number in the spectral space is consistent with that of $\left\langle u_{\theta}^{\prime} u_{\theta}^{\prime}\right\rangle^{+}$in the physical space shown in Fig. 5(b). Furthermore, the characteristic azimuthal length scale (or the spanwise separation) of the energetic streaky structures corresponding to the modal value of $k_{\theta}^{+} \tilde{E}_{\theta \theta}^{+}$also increases as the Reynolds number increases on both convex and concave cylinder sides of the concentric annular pipe.

From Fig. 17(a), case B1 barely captures the mode of $k_{\theta}^{+} \widetilde{E}_{\theta \theta}^{+}$on the convex side. This well explains the underprediction of $\left\langle u_{\theta}^{\prime} u_{\theta}^{\prime}\right\rangle^{+}$ on the convex side in Fig. 5(b). By contrast, the mode of the premultiplied $1 \mathrm{D}$ azimuthal spectrum $k_{\theta}^{+} \widetilde{E}_{\theta \theta}^{+}$is captured in cases B2-B5. However, the cutoff wavelengths of $k_{\theta}^{+} \tilde{E}_{\theta \theta}^{+}$differ. Specifically, at the cutoff wavelength, the magnitude of $k_{\theta}^{+} \widetilde{E}_{\theta \theta}^{+}$is $87.6 \%, 52.7 \%, 33.6 \%$, and $11.2 \%$ of its peak value on the convex side and $84.6 \%, 62.7 \%$, $20.1 \%$, and $7.0 \%$ of its peak value on the concave side in cases B2, B3, B4, and B5, respectively. In other words, based on the comparative study of $k_{\theta}^{+} \widetilde{E}_{\theta \theta}^{+}$at $r^{+}=30$ for the B-series test cases, the

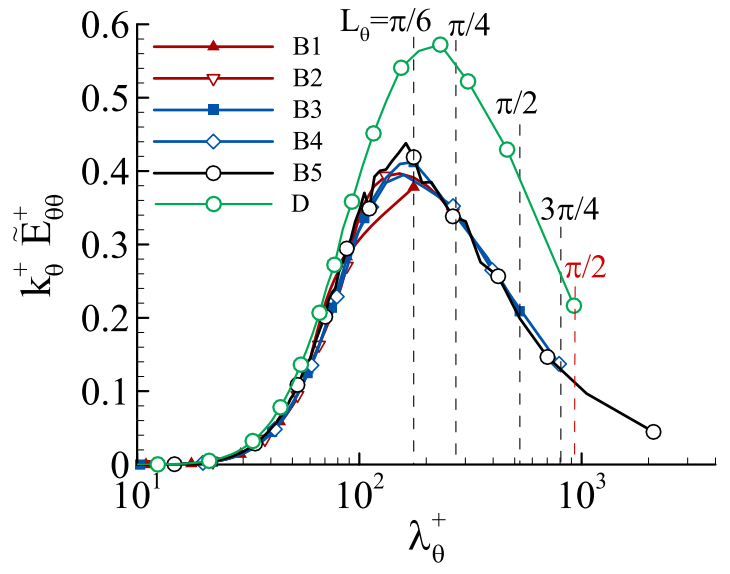

(a)

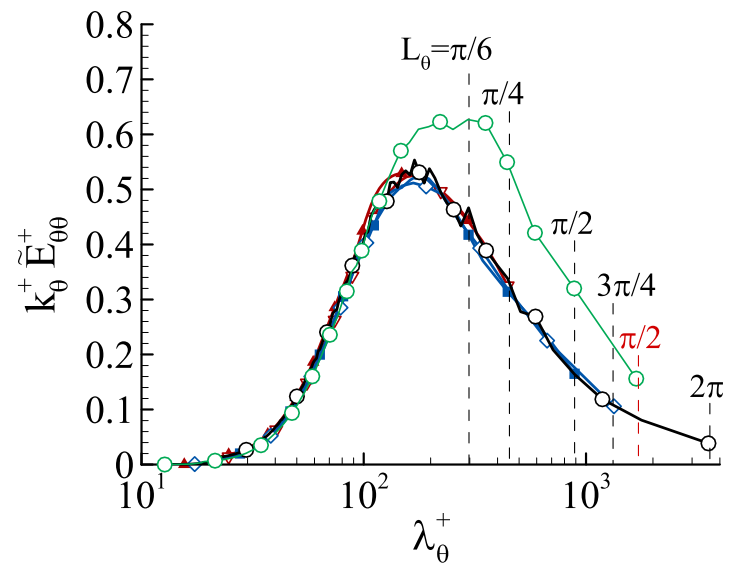

(b)

FIG. 17. Profiles of non-dimensionalized premultiplied 1D azimuthal energy spectra $k_{\theta}^{+} \widetilde{E}_{\theta \theta}^{+}$of the B-and D-series test cases at the cylindrical surface located at $r^{+}=30$ from the (a) convex and (b) concave walls. Vertical dashed lines demarcate the azimuthal computational sizes (i.e., $L_{\theta}$ values) for cases B1-B5 and D. 
computational domain size of $L_{\theta}=3 \pi / 4$ of case B4 resolves most of the energy-containing scales. Only a small portion of large scales remain unresolved that contain energy that is lower than $33.6 \%$ and $20.1 \%$ of the peak value of $k_{\theta}^{+} \widetilde{E}_{\theta \theta}^{+}$at the cutoff wavelength on the convex and concave sides of the concentric annular pipe, respectively. Furthermore, from Fig. 17, it is seen that as the nominal Reynolds number increases from $R e_{D_{h}}=8900$ to 17700 , the cutoff wavelength of case D takes place when the value of $k_{\theta}^{+} \widetilde{E}_{\theta \theta}^{+}$decays to $39 \%$ and $24 \%$ of its peak value on the convex and concave cylinder sides, respectively. This further indicates that the domain size of case D is suitable for capturing the characteristic azimuthal length scale of the energetic large-scale streaky structures at the higher nominal Reynolds number of $R e_{D_{h}}=17700$. Finally, it is very interesting to observe that in case $\mathrm{B} 5$, the value of $k_{\theta}^{+} \widetilde{E}_{\theta \theta}^{+}$decays to $11.2 \%$ and $7.0 \%$ of its peak value on the convex and concave sides of the concentric annular pipe, respectively. Considering that the azimuthal domain size of case B5 is a full circle with $L_{\theta}=2 \pi$, there is only one explanation that there are some low-energy flow structures in the flow, which have azimuthal periods larger than $2 \pi$. An example of this type of continuously developing azimuthal structure is the familiar helical structure.

\section{CONCLUSIONS}

The effect of computational domain size on the predictive accuracy of DNS results of a moderately curved turbulent concentric annular pipe flow has been studied in both physical and spectral spaces. In order to study the Reynolds number effects on the flow physics, two nominal Reynolds numbers are compared for $R e_{D_{h}}$ $=8900$ and 17700 . The effects of domain size on the flow field are investigated based on a comparative study of 12 test cases (including one reference case) at the lower nominal Reynolds number. If we solely focus on the mean velocity profiles, it would be very tempting to conclude that the axial and azimuthal domain sizes of $L_{z}=\pi \delta$ and $L_{\theta}=\pi / 6$ are sufficient for conducting DNS. However, based on an analysis of second-order statistical moments, it becomes apparent that the results of Reynolds stresses would not be accurate unless the minimum computational domain is extended to $L_{z}=6 \pi \delta$ and $L_{\theta}$ $=\pi / 4$. To determine if the computational domain is proper for capturing the length scales of energetic eddy motions, the $1 \mathrm{D}$ axial and azimuthal two-point correlation coefficients are examined, which indicate that the minimum computational domain needs to be kept at $L_{z}=6 \pi \delta$ and $L_{\theta}=\pi / 2$. The study of the minimum computational domain size for conducting DNS is further refined by investigating the characteristic wavelengths of turbulent flow structures in the spectral space. It is observed that an adequate computational domain that allows for capturing the most energetic eddy motions is that of case B4 (i.e., $L_{z}=12 \pi \delta$ and $L_{\theta}=3 \pi / 4$ ) based on the analysis of the $1 \mathrm{D}$ and $2 \mathrm{D}$ premultiplied spectra. Table III summarizes these results of minimum computational domains sizes required for accurately calculating turbulence statistics and for reproducing turbulence flow structures. As the nominal Reynolds number increases from $R e_{D_{h}}=8900$ and 17700 , the dimensional characteristic wavelengths of the most energetic streaky structures reduce, and in consequence, the minimum domain for properly performing DNS can be reduced to that of case $\mathrm{D}$ (i.e., $L_{z}=8 \pi \delta$ and $\left.L_{\theta}=\pi / 2\right)$.
TABLE III. Summary of the minimum computational domain sizes required for performing DNS for accurate calculations of different types of turbulence statistics at the nominal Reynolds number of $R e_{D_{h}}=8900$.

\begin{tabular}{lc}
\hline \hline $\begin{array}{l}\text { Criterion of } \\
\text { turbulence statistics }\end{array}$ & $\begin{array}{c}\text { Minimum domain } \\
\text { sizes (axial and } \\
\text { azimuthal) }\end{array}$ \\
\hline $\begin{array}{l}\text { Mean velocity } \\
\text { Reynolds stresses } \\
\text { Two-point correlations } \\
\text { 1D and 2D premultiplied } \\
\text { energy spectra }\end{array}$ & $L_{z}=\pi \delta$ and $L_{\theta}=\pi / 6$ \\
\hline \hline
\end{tabular}

In the current literature, there has been some disagreement on whether the radial position corresponding to the maximum velocity collocates with that of the zero mean shear stress in a turbulent concentric annular pipe flow. The inconsistency of the literature can now be concluded. Through a relatively simple approach, we show analytically that the radial positions of the maximum velocity and zero shear stress are, in fact, strictly collocated. This analytical result has been further validated using our DNS data.

Based on the aforementioned investigation of the minimum computational domain size, coherent flow structures of the concentric annular pipe flow are investigated at the lower nominal Reynolds number of $R e_{D_{h}}=8900$. The scales of energetic turbulent flow motions such as near-wall streaks and hairpin structures are visualized using instantaneous and conditionally averaged axial velocity fluctuations $\left(u_{z}^{\prime}\right)$, instantaneous axial vorticity $\left(\omega_{z}^{\prime+}\right)$, and swirling strength $\left(\lambda_{c i}\right)$. The scales of these structures are further studied by examining the $1 \mathrm{D}$ axial and azimuthal two-point correlation coefficients, and $1 \mathrm{D}$ and $2 \mathrm{D}$ premultiplied energy spectra. The $2 \mathrm{D}$ energy spectra show that both axial and azimuthal characteristic length scales of the near-wall streaks are larger on the convex side than on the concave side. This leads to an important conclusion that the challenge involved in accurately performing DNS of a concentric annular pipe flow mostly stems from the need of capturing large streaky structures on the convex side.

At a higher elevation of $\delta / 2$ away from the walls, hairpin packets are dominant energy-containing structures. The scales of these structures are similar on both convex and concave sides of the concentric annular pipe with a mode of approximately $\left(\lambda_{z}^{+}, \lambda_{\theta}^{+}\right)$ $=(405,190)$. The cycle of the generation of PHV from SHV and destruction of PHV into DHV is observed by showing a side view of hairpin packets. The pattern of generation and destruction of hairpin packets can be identified precisely by studying the axial mode of the $1 \mathrm{D}$ or $2 \mathrm{D}$ premultiplied spectrum of axial velocity fluctuations or vividly by displaying hairpin structures using the $\lambda_{c i}$ criterion. The characteristic wavelength of either PHV or SHV is approximately 400 wall units, and therefore, the axial separation of the hairpin necks (of PHV and SHV) is typically around 200 wall units. At $r^{+}=30$, there exist azimuthally oriented vortex filaments, which connect hairpin legs and are the most energetic around this elevation. These azimuthally oriented vortex filaments lay further away from the wall than the near-wall streaks, perturbed and pushed away by ejections to form hairpin arches at higher elevations. There 
is an interesting observation associated with the premultiplied $1 \mathrm{D}$ azimuthal energy spectrum $k_{\theta}^{+} \widetilde{E}_{\theta \theta}^{+}$of case B5: although the azimuthal domain size is a full circle with $L_{\theta}=2 \pi$, the value of $k_{\theta}^{+} \tilde{E}_{\theta \theta}^{+}$does not decay to zero at the cutoff wavelength (after one period). This indicates that there are some low-energy turbulence structures with azimuthal periods that are larger than $2 \pi$. Finally, it should be indicated that the number of DNS studies of concentric annular pipe flows is still rather limited in the current literature. The research findings reported in this paper are based on numerical simulations of flow in a concentric annular pipe of a fixed radius ratio. In order to develop a comprehensive understanding of this type of flow, a few more sub-topics can be considered in future studies, which include investigations of the aspect ratio effects on turbulent convection and the transport of Reynolds stresses in both physical and spectral spaces.

\section{ACKNOWLEDGMENTS}

The authors would like to thank Western Canada Research Grid (WestGrid) for access to supercomputing and storage facilities. Research funding from the Natural Sciences and Engineering Research Council (NSERC) of Canada to B.-C.W. is gratefully acknowledged.

\section{DATA AVAILABILITY}

The data that support the findings of this study are available from the corresponding author upon reasonable request.

\section{REFERENCES}

${ }^{1}$ G. I. Taylor, "Stability of a viscous liquid contained between two rotating cylinders," Philos. Trans. R. Soc., A 223(605-615), 289-343 (1923).

${ }^{2}$ G. I. Taylor, "Fluid friction between rotating cylinders I-Torque measurements," Proc. R. Soc. London, Ser. A 157(892), 546-564 (1936).

${ }^{3}$ S. Grossmann, D. Lohse, and C. Sun, "High-Reynolds number Taylor-Couette turbulence," Annu. Rev. Fluid Mech. 48, 53-80 (2016).

${ }^{4}$ J. G. Knudsen and D. L. Katz, "Velocity profiles in annuli," in Proceedings of the Midwestern Conference on Fluid Dynamics (Ann Arbor, MI, May 1950), pp. 175-203.

${ }^{5}$ J. A. Brighton and J. B. Jones, "Fully developed turbulent flow in annuli," J. Basic Eng. 86(4), 835-842 (1964).
${ }^{6} \mathrm{~K}$. Rehme, "Turbulent flow in smooth concentric annuli with small radius ratios," J. Fluid Mech. 64(2), 263-288 (1974).

${ }^{7}$ J. Nouri, H. Umur, and J. Whitelaw, "Flow of Newtonian and non-Newtonian fluids in concentric and eccentric annuli," J. Fluid Mech. 253, 617-641 (1993).

${ }^{8}$ S. Y. Chung, G. H. Rhee, and H. J. Sung, "Direct numerical simulation of turbulent concentric annular pipe flow: Part 1: Flow field," Int. J. Heat Fluid Flow 23(4), 426-440 (2002).

${ }^{9}$ S. Y. Chung and H. J. Sung, "Direct numerical simulation of turbulent concentric annular pipe flow: Part 2: Heat transfer," Int. J. Heat Fluid Flow 24(3), 399-411 (2003).

${ }^{10}$ B. J. Boersma and W.-P. Breugem, "Numerical simulation of turbulent flow in concentric annuli," Flow, Turbul. Combust. 86(1), 113-127 (2011).

${ }^{11} \mathrm{~J}$. Jiménez and P. Moin, "The minimal flow unit in near-wall turbulence," J. Fluid Mech. 225, 213-240 (1991).

${ }^{12}$ C. Chin, A. S. H. Ooi, I. Marusic, and H. M. Blackburn, "The influence of pipe length on turbulence statistics computed from direct numerical simulation data," Phys. Fluids 22(11), 115107 (2010).

${ }^{13}$ V. Avsarkison, S. Hoyas, M. Oberlack, and J. P. García-Galache, "Turbulent plane Couette flow at moderately high Reynolds number," J. Fluid Mech. 751, R1 (2014).

${ }^{14}$ Z. X. Yang and B.-C. Wang, "Capturing Taylor-Görtler vortices in a streamwiserotating channel at very high rotation numbers," J. Fluid Mech. 838, 658-689 (2018).

${ }^{15} \mathrm{M}$. Quadrio and P. Luchini, "Direct numerical simulation of the turbulent flow in a pipe with annular cross section," Eur. J. Mech.: B/Fluids 21(4), 413-427 (2002).

${ }^{16} \mathrm{H}$. Blackburn and S. J. Sherwin, "Formulation of a Galerkin spectral elementFourier method for three-dimensional incompressible flows in cylindrical geometries," J. Comput. Phys. 197(2), 759-778 (2004).

${ }^{17} \mathrm{~J}$. Kim, P. Moin, and R. Moser, "Turbulence statistics in fully developed channel flow at low Reynolds number," J. Fluid Mech. 177, 133-166 (1987).

${ }^{18} \mathrm{R}$. Adrian, "Hairpin vortex organization in wall turbulence," Phys. Fluids 19(4), 041301 (2007).

${ }^{19}$ R. D. Moser, J. Kim, and N. N. Mansour, "Direct numerical simulation of turbulent channel flow up to $R e_{\tau}=590$," Phys. Fluids 11, 943-945 (1999).

${ }^{20}$ S. I. Chernyshenko and M. F. Baig, "The mechanism of streak formation in nearwall turbulence," J. Fluid Mech. 544, 99-131 (2005).

${ }^{21}$ R. Adrian, "Stochastic estimation of conditional structure: A review," Appl. Sci. Res. 53(3-4), 291-303 (1994).

${ }^{22} \mathrm{X}$. Wu, J. R. Baltzer, and R. Adrian, "Direct numerical simulation of a 30R long turbulent pipe flow at $R^{+}=685$ : Large-and very large-scale motions," J. Fluid Mech. 698, 235-281 (2012).

${ }^{23}$ J. P. Monty, J. A. Stewart, R. C. Williams, and M. S. Chong, "Large-scale features in turbulent pipe and channel flows," J. Fluid Mech. 589, 147-156 (2007). 